NANYANG

TECHNOLOGICAL

UNIVERSITY
Division of Economics, EGC School of Humanities and Social Sciences

Nanyang Technological University 14 Nanyang Drive Singapore 637332

\title{
Efficiency Gains by Modifying GMM Estimation in Linear Models under Heteroskedasticity
}

\section{Jan F. KIVIET and Qu FENG}

10 November 2014

EGC Report No: 2014/13

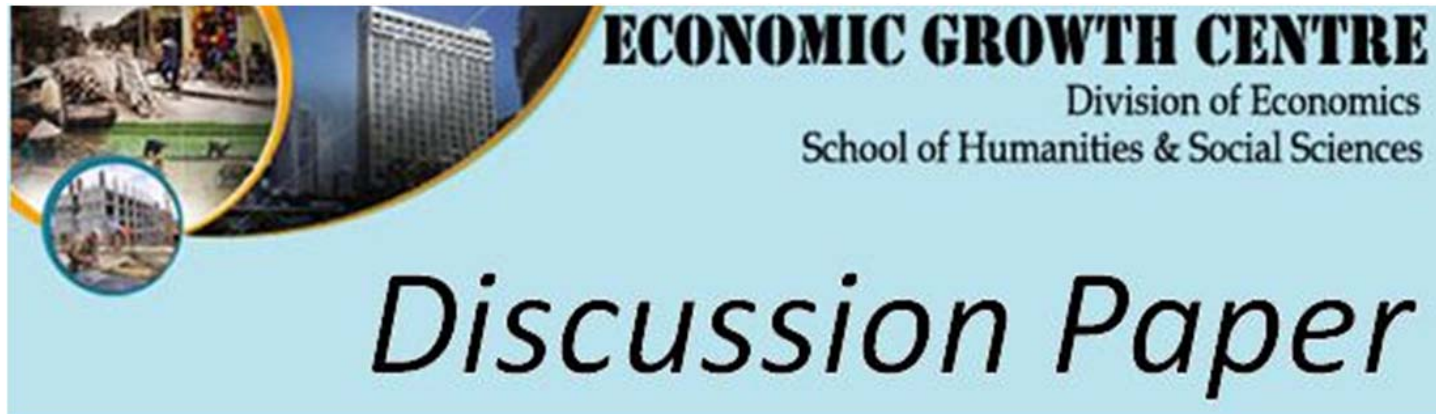


The author(s) bear sole responsibility for this paper.

Views expressed in this paper are those of the author(s) and not necessarily those of the Economic Growth Centre, NTU. 


\title{
Efficiency Gains by Modifying GMM Estimation in Linear Models under Heteroskedasticity
}

\author{
Jan F. Kiviet* and Qu Feng ${ }^{\dagger}$
}

Version of 10 November $2014^{\ddagger}$

JEL-code: C01, C13, C26.

Keywords: efficiency, generalized method of moments, instrument strength, non-spherical disturbances, (un) conditional moment assumptions

\begin{abstract}
While coping with nonsphericality of the disturbances, standard GMM suffers from a blind spot for exploiting the most effective instruments when these are obtained directly from unconditional rather than conditional moment assumptions. For instance, standard GMM counteracts that exogenous regressors are used as their own optimal instruments. This is easily seen after transmuting GMM for linear models into IV in terms of transformed variables. It is demonstrated that modified GMM (MGMM), exploiting straight-forward modifications of the instruments, can achieve substantial efficiency gains and bias reductions, even under mild heteroskedasticity. Feasible MGMM implementations and their standard error estimates are examined and compared with standard GMM and IV for a range of typical models for cross-section data, both by simulation and by empirical illustration.
\end{abstract}

\footnotetext{
*Emeritus Professor of Econometrics, Amsterdam School of Economics, University of Amsterdam, Valckenierstraat 65, 1018 XE Amsterdam, The Netherlands (j.f.kiviet@uva.nl) and Visting Professor at the Division of Economics, School of Humanities and Social Sciences, Nanyang Technological University, 14 Nanyang Drive, Singapore 637332 (jfkiviet@ntu.edu.sg).

$\dagger^{\dagger}$ Division of Economics, School of Humanities and Social Sciences, Nanyang Technological University, 14 Nanyang Drive, Singapore 637332 (qfeng@ntu.edu.sg).

${ }^{\ddagger}$ We would like to thank Manuel Arellano, Paul Bekker, Joerg Breitung, Geert Dhaene, Guido Kuersteiner, Choon-Geol Moon, Liangjun Su, Tom Wansbeek and participants at various seminars and conferences for their comments and suggestions on earlier versions of this study.
} 


\section{Introduction}

For over three decades GMM (generalized method of moments) excels as the generic orthogonality conditions based optimal technique for semiparametric estimation. It subsumes the majority of linear and nonlinear econometric estimators. Not starting off from tight fully parametric distributional assumptions, consequently GMM may be surpassed in efficiency by other methods under more specific conditions, but - as a limited information technique - it has the attraction of exhibiting robustness over a wider set of situations. Therefore, applications of GMM are numerous, especially for the analysis of continuously varying dependent variables both in microeconometric studies of crosssectional or panel data models and in the macroeconometric analysis of time-series, in particular when stationarity assumptions can be made.

The status of GMM seems undisputable and is warranted by its following achievement: when the variance matrix of the adopted moment functions can be estimated consistently and these moment conditions imply a sufficient number of instrumental variables then the standard GMM technique of Hansen (1982) deals optimally with both any nonsphericality of the disturbances and any overidentification, given the actual set of instrumental variables that is being used. However, as discussed in Newey (1993) and Arellano (2003a,b), this does not necessarily imply that the moment conditions as such are being exploited optimally. The instrumental variables are usually obtained directly from unconditional moment conditions, whereas actually more comprehensive conditional moment conditions may hold. These allow to exploit as instruments also non-trivial transformations of instrumental variables. We will show that especially in models with heteroskedasticity this means that first transforming the model such that its disturbances become homoskedastic leads to orthogonality conditions with respect to these transformed disturbances from which much stronger instruments may emerge. These will not only lead to reduced bias and variance, but also to a better correspondence between actual distribution in finite samples and its asymptotic approximation. This all supports a strategy for finding instrumental variables close to the original IV (instrumental variables) approach suggested by Sargan $(1958,1959) .{ }^{1}$

For the sake of simplicity we will focus here on both unfeasible and feasible standard and modified implementations of GMM just in linear models with heteroskedasticity. However, the results will have obvious implications for more general models too. The possibility to replace relatively weak instruments by stronger instruments is of great interest, because weakness of instruments has serious consequences for inference. This has been extensively investigated for more than two decades already (see Staiger and Stock, 1997, and Andrews and Stock, 2007, and its references). However, this literature is largely confined to IV or 2SLS (two-stage least-squares) estimation of linear models with i.i.d. (independently and identically distributed) errors. It has not really been extended yet to the case of GMM. Below, however, we show that for linear models with nonspherical disturbances all aspects of GMM can in principle be understood in terms of IV applied to a model with spherical disturbances in transformed variables. Since in this transmutation the instruments are affected in a topsy-turvy manner, the correspondence in linear regression between GMM and IV is less straight-forward than in the similar but more basic case of GLS (generalized least-squares), which is equivalent

\footnotetext{
${ }^{1}$ For an overview which puts the IV and GMM approaches into historical perspective see Arellano (2002) and for a monograph on GMM see Hall (2005).
} 
to OLS (ordinary least-squares) applied to transformed variables. We will demonstrate that under heteroskedasticity the reverse transformation of the instruments in standard GMM is detrimental for their strength. But, we will also show that this can easily be prevented by implementing GMM in a modified way.

For the simple (though unrealistic) case of known heteroskedasticity the standard and modified forms of GMM that we consider build on exactly the same moment conditions. However, from these they extract different instrumental variables. To find out what the major practical consequences are requires some well-designed simulation investigations. When performing these we exploit the in practice unavailable optimal weighting matrix, because our first interest is in finding out what the actual potential efficiency gains are of modified GMM. Next, we also focus on feasible implementations and learn that a fully nonparametric implementation of our modified estimator is ineffective. However, a parametric setup, where use is made of a proper specification of the determining factors of the (conditional) heteroskedasticity, yields substantial efficiency gains in comparison with the unfeasible optimal standard GMM estimator. We find that under nonextreme circumstances root mean squared errors may reduce by about $50 \%$. In this context the distinction between conditional and unconditional moment assumptions proves to be crucial. Adopting just unconditional moment assumptions yields the standard instrumental variables. Assuming that conditional moment assumptions hold, allows to transform the instruments by weights inspired by the skedastic function. When this can be estimated consistently one can employ instruments which asymptotically attain the efficiency bound achieved by the unfeasible optimal instruments.

In this paper the focus is the (in)efficiency of IV and of (modifications of) GMM under heteroskedasticity. A similar analysis regarding serial correlation (the major worry in Sargan's approach) is left for future research. Our analysis of possibilities to improve on the efficiency of the standard implementation of GMM under heteroskedasticity leads to the conclusion that in practice one should aim to weigh observations first in order to get as close to homoskedasticity as possible. Not before, but after that (as already done by some researchers on sensible intuitive grounds), one should design a matrix of instruments according to the adopted orthogonality conditions in terms of the weighted variables. In any next GMM iterations the required transformation of variables should have deliberate implications for the chosen transformation of the instruments. Similar conclusions have been drawn at various places in the literature without explicit reference to GMM, see Bowden and Turkington (1984), White (1986), Baltagi and Li (1992), Wooldridge (2010 p.351, 2013 p.516) and Iglesias and Phillips (2012). Models with endogenous regressors and heteroskedasticity have also been considered in another line of research (for a recent contribution see the references in and the study by Hausman et al., 2012), but there the purpose is not to improve estimator efficiency, but to make the variance estimates of suboptimal estimators robust in the presence of heteroskedasticity.

The structure of this study is as follows. In section 2 we show how GMM can be interpreted in terms of transformed IV, which immediately demonstrates that in the presence of heteroskedasticity it leads in principle to using unnecessarily weak instruments. It also suggests an unfeasible alternative GMM estimator. Section 3 focusses on obtaining optimal instruments and produces a parametric and a nonparametric feasible implementation of a modified GMM estimator. It also discusses the estimation of the variance of coefficient estimators. Section 4 presents a Monte Carlo design for a typical family of simultaneous heteroskedastic cross-section models followed in Section 5 by 
simulation results which demonstrate the huge potential efficiency gains by modifying GMM as well as the difficulties to realize these by feasible implementations. In Section 6 alternative implementations of feasible GMM are applied to empirical data to illustrate the practical consequences of the suggested modifications. Section 7 concludes.

\section{GMM in linear models and its relation to IV}

We consider $n$ sample observations of the single linear regression model $y_{i}=x_{i}^{\prime} \beta+\varepsilon_{i}$ where $i=1, \ldots, n$. This model can be written compactly as

$$
y=X \beta+\varepsilon,
$$

where the $n \times K$ regressor matrix $X=\left(x_{1} \ldots x_{n}\right)^{\prime}$ is supposed to have full column rank. Some regressors may be endogenous, hence possibly $E\left(x_{i} \varepsilon_{i}\right) \neq 0$. Available are also $L$ variables $z_{i}$ which provide an identifying set of unconditional moment assumptions

$$
E\left(z_{i} \varepsilon_{i}\right)=0
$$

where $Z=\left(z_{1} \ldots z_{n}\right)^{\prime}$ is an $n \times L$ matrix of rank $L \geq K$. Provided $Z^{\prime} X$ has rank $K$ the IV (or 2SLS) estimator is given by

$$
\hat{\beta}_{I V}=\left[X^{\prime} Z\left(Z^{\prime} Z\right)^{-1} Z^{\prime} X\right]^{-1} X^{\prime} Z\left(Z^{\prime} Z\right)^{-1} Z^{\prime} y .
$$

By defining for any full column rank matrix $A$ the projection matrix $P_{A}=A\left(A^{\prime} A\right)^{-1} A^{\prime}$ the expression for $\hat{\beta}_{I V}$ can be condensed to $\left(X^{\prime} P_{Z} X\right)^{-1} X^{\prime} P_{Z} y$, and even further, by denoting the fitted first-stage regression results as $\hat{X}=P_{Z} X$, to the so-called second-stage OLS regression $\left(\hat{X}^{\prime} \hat{X}\right)^{-1} \hat{X}^{\prime} y$. The IV estimator is consistent and when $\varepsilon_{i} \sim \operatorname{iid}\left(0, \sigma_{\varepsilon}^{2}\right)$ has limiting distribution

$$
n^{1 / 2}\left(\hat{\beta}_{I V}-\beta\right) \stackrel{d}{\rightarrow} N\left(0, \sigma_{\varepsilon}^{2} \operatorname{plim} n\left(X^{\prime} P_{Z} X\right)^{-1}\right),
$$

provided sufficient further regularity conditions hold.

If $\varepsilon \sim(0, \Sigma)$, with $\Sigma$ a symmetric positive definite matrix, while (2.2) still holds, then the preferred estimator of $\beta$ is obtained by GMM, given by

$$
\hat{\beta}_{G M M}=\left[X^{\prime} Z\left(Z^{\prime} \Omega Z\right)^{-1} Z^{\prime} X\right]^{-1} X^{\prime} Z\left(Z^{\prime} \Omega Z\right)^{-1} Z^{\prime} y,
$$

where $\Omega$ can be any non-zero scalar multiple of $\Sigma$. It is convenient to define

$$
\Omega=\Sigma / \sigma_{\varepsilon}^{2} \text { with } \sigma_{\varepsilon}^{2}=\operatorname{tr}(\Sigma) / n \text {, so that } \operatorname{tr}(\Omega)=n .
$$

The GMM estimator is optimal in the sense that in aiming to bring the $L$ sample moments $n^{-1} Z^{\prime}\left(y-X \hat{\beta}_{G M M}\right)$ jointly as closely as possible to zero, it weighs them in a quadratic form in such a way that the variance of its limiting distribution

$$
n^{1 / 2}\left(\hat{\beta}_{G M M}-\beta\right) \stackrel{d}{\rightarrow} N\left(0, \operatorname{plim} n\left[X^{\prime} Z\left(Z^{\prime} \Sigma Z\right)^{-1} Z^{\prime} X\right]^{-1}\right)
$$

is minimal in a matrix sense. GMM simplifies to IV when $\Sigma=\sigma_{\varepsilon}^{2} I$ or $\Omega=I$.

In the special case $L=K$ the matrix $Z^{\prime} X$ is invertible and, irrespective of the value of $\Omega$, we obtain

$$
\hat{\beta}_{G M M}=\left(Z^{\prime} X\right)^{-1} Z^{\prime} y=\hat{\beta}_{I V} .
$$


Then all exploited moment conditions can be satisfied in the sample, so that the weighting matrix has no effect, and therefore $\Omega$ cannot play a role in optimizing the estimator. Of course, it still affects the variance of its limiting distribution, which is $\sigma_{\varepsilon}^{2} \operatorname{plim} n\left(Z^{\prime} X\right)^{-1} Z^{\prime} \Omega Z\left(X^{\prime} Z\right)^{-1}$ when $L=K$.

When the regressors are such that $E\left(x_{i} \varepsilon_{i}\right)=0$ they can all be used as instruments. Then substituting $X$ for $Z$ we find, also when $\Omega \neq I$,

$$
\hat{\beta}_{G M M}=\left(X^{\prime} X\right)^{-1} X^{\prime} y=\hat{\beta}_{O L S} .
$$

However, it is well know that in that case the optimal estimator is

$$
\hat{\beta}_{G L S}=\left(X^{\prime} \Omega^{-1} X\right)^{-1} X^{\prime} \Omega^{-1} y .
$$

Apparently, the optimality of GMM is not universal. We will clarify this disturbing result $^{2}$ below, but first we shall demonstrate that in linear models GMM is equivalent to applying IV to a properly transformed model upon exploiting instruments which are also subjected to a transformation related to - but different from - the model transformation.

It is well-known that the GLS estimator can be obtained by applying OLS to a suitably transformed model. This purely algebraic result is demonstrated as follows. Let $\Omega^{-1}=\Psi^{\prime} \Psi$, where $\Psi$ has full rank but is generally non-unique. Premultiplication of (2.1) by $\Psi$ yields the transformed model

$$
y^{*}=X^{*} \beta+\varepsilon^{*},
$$

where $y^{*}=\Psi y, X^{*}=\Psi X$ and $\varepsilon^{*}=\Psi \varepsilon \sim\left(0, \sigma_{\varepsilon}^{2} \Psi \Omega \Psi^{\prime}\right)$ with $\Psi \Omega \Psi^{\prime}=\Psi\left(\Psi^{\prime} \Psi\right)^{-1} \Psi^{\prime}=I$. Applying OLS to this transformed model yields

$$
\left(X^{* \prime} X^{*}\right)^{-1} X^{* \prime} y^{*}=\left(X^{\prime} \Psi^{\prime} \Psi X\right)^{-1} X^{\prime} \Psi^{\prime} \Psi y=\hat{\beta}_{G L S} .
$$

Now consider estimating transformed model (2.10) by IV, while using the instruments

$$
Z^{\dagger}=\left(\Psi^{\prime}\right)^{-1} Z
$$

These are such that $Z^{\dagger \prime} \varepsilon^{*}=Z^{\dagger \prime} \Psi \varepsilon=Z^{\prime} \varepsilon$, and thus they exploit still exactly the original moment conditions. Because $\varepsilon^{*} \sim\left(0, \sigma_{\varepsilon}^{2} I\right)$ we may estimate the transformed model by IV, which yields

$$
\left(X^{* \prime} P_{Z^{\dagger}} X^{*}\right)^{-1} X^{* \prime} P_{Z^{\dagger}} y^{*}=\left[X^{\prime} \Psi^{\prime} P_{Z^{\dagger}} \Psi X\right]^{-1} X^{\prime} \Psi^{\prime} P_{Z^{\dagger}} \Psi y=\hat{\beta}_{G M M},
$$

where we used $P_{Z^{\dagger}}=\left(\Psi^{\prime}\right)^{-1} Z\left(Z^{\prime} \Omega Z\right)^{-1} Z^{\prime} \Psi^{-1}$. Hence, GMM corresponds algebraically to applying IV to a "GLS-like" transformed model, exploiting instruments which are transformed by a linear transformation which is actually the inverse of the transpose of the model transformation.

Now consider the special case in which $X^{*}$ and $\varepsilon^{*}$ are such that $E\left(x_{i}^{*} \varepsilon_{i}^{*}\right)=0$. Of course, then one should estimate the transformed model by OLS (which conforms to using GLS in the untransformed model) and not by IV while using as instruments $X^{\dagger}=\left(\Psi^{\prime}\right)^{-1} X$. However, the latter is what standard GMM prescribes and it conforms to applying suboptimal OLS to the untransformed model. The handicap of standard

\footnotetext{
${ }^{2}$ Our clarification is less abstract than those presented by Davidson and MacKinnon (2004, p.358), Cameron and Trivedi (2005, p.747) and Wooldridge (2010, p.542).
} 
GMM is that it lacks the flexibility to replace the instruments $Z$ by $\Omega^{-1} Z$ when these happen to be valid ${ }^{3}$ too. The above demonstrates that we better should not decide upon which variables to use as instruments by considering the regressors of the untransformed model, but first do the transformation and next find appropriate (valid and as strong as possible) instruments for the regressors of the transformed model. That this is also the case in models with some endogenous regressors is illustrated by the following.

Assume that $X=\left(X_{1} X_{2}\right)$, where $X_{1}$ contains $K_{1} \geq 0$ exogenous regressors, and $X_{2}$ contains $K_{2} \geq 0$ possibly endogenous regressors, hence $E\left(\varepsilon \mid X_{1}\right)=0$ thus $E\left(X_{1}^{\prime} \varepsilon\right)=0$, whereas $E\left(X_{2}^{\prime} \varepsilon\right)$ is unknown. Of course $K=K_{1}+K_{2}>0$, so $X_{1}$ and $X_{2}$ can be void, but not both at the same time. We consider the case where $K_{1}>0$. Because $E(\varepsilon \mid$ $\left.X_{1}\right)=0$ implies $E\left(\varepsilon^{*} \mid X_{1}^{*}\right)=E\left(\Psi \varepsilon \mid \Psi X_{1}\right)=0$ it is obvious that when estimating the transformed model (2.10) by IV we should preferably include $X_{1}^{*}$ in the matrix of instruments. However, standard GMM would include $X_{1}$ in the matrix of instruments for the untransformed model, implying that $X_{1}^{\dagger}=\left(\Psi^{\prime}\right)^{-1} X_{1}$ is a component of the instrument matrix it employs to the transformed model. However, it is unlikely that in the first-stage regression of $X^{*}$ on the instruments $Z^{\dagger}=\left(X_{1}^{\dagger} Z_{2}^{\dagger}\right)$ a perfect fit will be realized for $X_{1}^{*}$, whereas this occurs when including $X_{1}^{*}$ in the instrument set for the transformed model. Standard GMM does not automatically use regressors which are exogenous in the transformed model as instruments for estimating the transformed model. The optimality of standard GMM is achieved over the sample moment conditions expressed in terms of the chosen instruments $Z$. The above illustrates that efficiency gains seem possible by allowing to consider transformations of $Z$, by pre-multiplying $Z$ with an $n \times n$ matrix, as well. Of course, such transformations should still be in agreement with the adopted population moment conditions. ${ }^{4}$

When $\Omega$ is diagonal, say $\Omega=\operatorname{diag}\left(\omega_{1}, \ldots, \omega_{n}\right)$ with $\sum_{i=1}^{n} \omega_{i}=n$ and all $\omega_{i}>0$, then the moment conditions $E\left(z_{i} \varepsilon_{i}\right)=0$ are equivalent with $E\left(z_{i} \varepsilon_{i} / \omega_{i}\right)=0$. Hence, a possible modification of GMM is using instrument matrix $\Omega^{-1} Z$, rather than $Z$. This yields the modified GMM estimator

$$
\hat{\beta}_{M G M M}=\left[X^{\prime} \Omega^{-1} Z\left(Z^{\prime} \Omega^{-1} Z\right)^{-1} Z^{\prime} \Omega^{-1} X\right]^{-1} X^{\prime} \Omega^{-1} Z\left(Z^{\prime} \Omega^{-1} Z\right)^{-1} Z^{\prime} \Omega^{-1} y
$$

with limiting distribution

$$
n^{1 / 2}\left(\hat{\beta}_{M G M M}-\beta\right) \stackrel{d}{\rightarrow} N\left(0, \sigma_{\varepsilon}^{2} \operatorname{plim} n\left[X^{\prime} \Omega^{-1} Z\left(Z^{\prime} \Omega^{-1} Z\right)^{-1} Z^{\prime} \Omega^{-1} X\right]^{-1}\right) .
$$

MGMM $^{5}$ would be more efficient than GMM when the difference between the matrices plim $n^{-1} X^{* \prime} P_{Z^{*}} X^{*}$ and plim $n^{-1} X^{* \prime} P_{Z^{\dagger}} X^{*}$ is positive (semi-)definite. This seems likely to occur, when $Z$ is chosen deliberately (as is usually the case) such that it yields good fits when regressing the columns of $X$ on $Z$, because that should also lead to a similar good fit when regressing $X^{*}$ on $Z^{*}$. The latter fit seems likely to be better than for the regressions of the columns of $X^{*}$ on $Z^{\dagger}$, because here the weights applied to the rows of the $X^{*}$ are the opposite of the weights applied to the corresponding rows of $Z^{\dagger}$.

\footnotetext{
${ }^{3}$ When we speak of a valid instrument this refers to the validity of the corresponding orthogonality condition. The degree by which a (not necessarily valid) instrument is effective (or not) with respect to achieving attractive estimator spread (and possibly also location) will always be addressed here by instrument strength (or weakness).

${ }^{4}$ As is well-know, IV and GMM are invariant regarding transformations by post-multiplying $Z$ by a nonsingular $L \times L$ matrix. Here we are examining the effects of transformations by pre-multiplying $Z$.

${ }^{5}$ Bowden and Turkington (1984, p.69) consider this estimator, but suppose it to be of limited value.
} 
However, MGMM is likely to be worse than GMM when GMM is implemented in a clever way by using instruments $\Omega^{-1} Z$ for the untransformed model because then it actually yields MGMM of (2.13), whereas substituting $\Omega^{-1} Z$ for $Z$ in MGMM would now involve IV estimation of the transformed model using the rather awkward instruments $\Omega^{-3 / 2} Z$. This shows that the problem with GMM that we are highlighting here can be avoided simply by employing GMM in a more sophisticated way than is standard, namely by choosing the instruments less naively from the initially adopted moment conditions and next adjusting them in the light of the established heteroskedasticity. In the special case $L=K$, where the expression for the standard GMM estimator is invariant with respect to $\Omega$ and simplifies to $\left(Z^{\prime} X\right)^{-1} Z^{\prime} y$, the MGMM estimator yields $\left(Z^{\prime} \Omega^{-1} X\right)^{-1} Z^{\prime} \Omega^{-1} y$, which is efficient when $Z=X$.

To use the above results on MGMM directly for inference on $\beta$ would require that the numerical values of all the elements of the matrix $\Omega$ are known, which is usually not the case. In the next section we will aim at actual gains in efficiency by designing operational implementations of modifications of GMM in situations where the values of the diagonal elements of $\Omega$ are unknown and all nondiagonal elements are zero. We will examine both nonparametric and parametric approaches towards coping efficiently with heteroskedasticity and simultaneity jointly.

\section{Optimality and efficiency under heteroskedasticity}

In (2.2) we made the $L$ unconditional moment or orthogonality assumptions $E\left(z_{i} \varepsilon_{i}\right)=0$, which suggest using instrumental variable matrix $Z$. But, from (2.2) it easily follows that $E\left(z_{i} \varepsilon_{i} / \omega_{i}\right)=0$ too. Thus, assuming for the moment that $\Omega$ is known, using instrument matrix $\Omega^{-1} Z$ is possible too. For element $z_{i l}$ of $z_{i}$, where $l \in\{1, \ldots, L\}$, we would usually also have $E\left(z_{i l}^{2} \varepsilon_{i}\right)=0$. In fact, any nonlinear deterministic function of $z_{i l}$ would in principle provide an extra valid instrument, yielding an abundance of instruments. If instead of (2.2), we could make the conditional moment assumption

$$
E\left(\varepsilon_{i} \mid z_{i}\right)=0,
$$

then for all elements of $z_{i}$ unconditional moment assumptions of the form

$$
E\left[g\left(z_{i l}\right) \varepsilon_{i}\right]=0, l=1, \ldots, L
$$

hold, where $g(\cdot)$ is a vector function of distinct scalar deterministic functions. The number of elements of vector $g\left(z_{i l}\right)$ could be infinitely large. Conditional moment assumption (3.1) implies (2.2), but not the other way around. Assumption (2.2) is weaker, but in practice it is usually the case that when $E\left(z_{i l} \varepsilon_{i}\right)=0$ holds, it is hard to find arguments why unconditional moment conditions of the form (3.2) will not hold either. In other words, when we have $L$ valid instruments in $Z$, usually we can easily produce many many more. However, not all of these will be very strong. We would like to be able to assemble those which are optimal in some sense.

Newey (1993) and Arellano (2003a, Appendix B) address this issue in a setup, where $\left(y_{i}, x_{i}^{\prime}, z_{i}^{\prime}\right)$ are i.i.d. and the conditional moment assumption $E\left(y_{i}-x_{i}^{\prime} \beta \mid z_{i}\right)=0$ is made. Then the optimal instruments ${ }^{6}$ are shown to be given by the $K \times 1$ vector

$$
\bar{g}\left(z_{i}\right)=E\left(x_{i} \mid z_{i}\right) / E\left(\varepsilon_{i}^{2} \mid z_{i}\right) .
$$

\footnotetext{
${ }^{6}$ see also Cameron and Trivedi (2005, p.188).
} 
The corresponding unfeasible optimal (UO) GMM estimator uses $\bar{g}\left(z_{i}\right)$ as instruments. It has asymptotic variance

$$
V_{U O}=\left\{E\left(x_{i} \mid z_{i}\right)\left[E\left(\varepsilon_{i}^{2} \mid z_{i}\right)\right]^{-1} E\left(x_{i}^{\prime} \mid z_{i}\right)\right\}^{-1}=E\left(\varepsilon_{i}^{2} \mid z_{i}\right)\left\{\bar{g}\left(z_{i}\right) \bar{g}\left(z_{i}\right)^{\prime}\right\}^{-1} .
$$

Newey (1993) shows that this variance $V_{U O}$ is a lower bound for the asymptotic variance of all IV/GMM estimators for the present model, exploiting (3.1). When the instruments $z_{i}$ contain some exogenous elements of $x_{i}$, so $x_{i}=\left(x_{1 i}^{\prime}, x_{2 i}^{\prime}\right)^{\prime}$ and $z_{i}=\left(x_{1 i}^{\prime}, z_{2 i}^{\prime}\right)^{\prime}$, then

$$
E\left(x_{i}^{\prime} \mid z_{i}\right)=\left(x_{1 i}^{\prime} E\left(x_{2 i}^{\prime} \mid z_{i}\right)\right)
$$

which indicates that the optimal instrument matrix should have a component proportional to $\Omega^{-1} X_{1}$, which vindicates our earlier more informal derivations.

The optimal instruments for the linear model under conditions $E\left(\varepsilon_{i} \mid z_{i}\right)=0$ are also discussed in Davidson and MacKinnon (2004, Chapter 9) for dependent data. They show that the matrix of optimal instruments is given by $\sigma_{\varepsilon}^{-2} \Omega^{-1} \bar{X}$, where $\bar{X}=\left(\bar{x}_{1}, \cdots, \bar{x}_{n}\right)^{\prime}$ and $\bar{x}_{i}$ is defined as $E\left(x_{i} \mid \Xi_{i}\right)$, where $\Xi_{i}$ contains the set of all deterministic functions of the elements of $\left(z_{i}, \ldots, z_{1}\right)$. It easily follows that an expression for the asymptotic variance of the GMM estimator using these optimal instruments is

$$
\sigma_{\varepsilon}^{2} \operatorname{plim} n\left(\bar{X}^{\prime} \Omega^{-1} \bar{X}\right)^{-1}
$$

which corresponds to (3.4). Since $\bar{X}$ is not observable, Davidson and MacKinnon (2004, p.361) suggest to use instruments $\Omega^{-1} Z$. When the span of $\bar{X}$ is a subset of that of $Z$, the GMM estimator using instruments $\Omega^{-1} Z$ has the same asymptotic variance, implying that $\Omega^{-1} Z$ should be used as optimal instrument matrix. This estimator is only feasible when $\Omega$ is known.

When $\Omega$ is unknown, we could - as in feasible GLS - parametrize the functional form of the skedastic function, for instance as $\sigma_{i}=\sigma_{\varepsilon}^{2} \omega_{i}=h\left(z_{i}^{\prime} \gamma\right)$, where $\sigma_{i}$ and $\omega_{i}$ represent the $i$-th diagonal element of the matrix $\Sigma$ and $\Omega$ respectively. From consistent estimates $\hat{\sigma}_{i}=h\left(z_{i}^{\prime} \hat{\gamma}\right)$ a consistent estimate $\hat{\Sigma}$ can be obtained. This enables to obtain as an alternative for the unfeasible estimator (2.5), the standard feasible parametric GMM estimator

$$
\hat{\beta}_{F p G M M}=\left[X^{\prime} Z\left(Z^{\prime} \hat{\Sigma} Z\right)^{-1} Z^{\prime} X\right]^{-1} X^{\prime} Z\left(Z^{\prime} \hat{\Sigma} Z\right)^{-1} Z^{\prime} y,
$$

and as an alternative to (2.13) the feasible parametric MGMM estimator, which uses the instruments $\hat{\Sigma}^{-1} Z$ to the untransformed model, and is given by

$$
\hat{\beta}_{F p M G M M}=\left[X^{\prime} \hat{\Sigma}^{-1} Z\left(Z^{\prime} \hat{\Sigma}^{-1} Z\right)^{-1} Z^{\prime} \hat{\Sigma}^{-1} X\right]^{-1} X^{\prime} \hat{\Sigma}^{-1} Z\left(Z^{\prime} \hat{\Sigma}^{-1} Z\right)^{-1} Z^{\prime} \hat{\Sigma}^{-1} y .
$$

Here $E\left(\varepsilon_{i}^{2}\right)=h\left(z_{i}^{\prime} \gamma\right)$ establishes a moment condition that is nonlinear in $\beta$, which could be exploited directly when deriving a method of moments estimator. In our implementations, however, we use a simple 2-step procedure, in which $\hat{\gamma}$ is obtained by regressing $h^{-1}\left(\hat{\varepsilon}_{i}^{2}\right)$ on $z_{i}$, where $\hat{\varepsilon}_{i}=y_{i}-x_{i}^{\prime} \hat{\beta}_{I V}$ is consistent for the disturbance $\varepsilon_{i}$.

Alternatively, the case of unknown $\Omega$ can also be tackled nonparametrically. For standard GMM and unspecified heteroskedasticity the most basic feasible procedure, which is asymptotically equivalent to unfeasible GMM, is another 2-step procedure. Here the IV residuals $\hat{\varepsilon}_{i}$ are used to obtain a consistent estimator for $Z^{\prime} \Sigma Z / n$ by $\hat{S}_{z z}=$ $n^{-1} \sum_{i=1}^{n} \hat{\varepsilon}_{i}^{2} z_{i} z_{i}^{\prime}$ and the second step is

$$
\hat{\beta}_{F n p G M M}=\left[X^{\prime} Z \hat{S}_{z z}^{-1} Z^{\prime} X\right]^{-1} X^{\prime} Z \hat{S}_{z z}^{-1} Z^{\prime} y
$$


Inspired by this, a 2-step feasible nonparametric MGMM estimator can be calculated on the basis of

$$
\hat{\beta}_{F n p M G M M}=\left[\hat{Q}_{z x}^{\prime} \hat{Q}_{z z}^{-1} \hat{Q}_{z x}\right]^{-1} \hat{Q}_{z x}^{\prime} \hat{Q}_{z z}^{-1} \hat{Q}_{z y},
$$

where $\hat{Q}_{z z}=n^{-1} \sum_{i=1}^{n} \hat{\varepsilon}_{i}^{-2} z_{i} z_{i}^{\prime}, \hat{Q}_{z x}=n^{-1} \sum_{i=1}^{n} \hat{\varepsilon}_{i}^{-2} z_{i} x_{i}^{\prime}$ and $\hat{Q}_{z y}=n^{-1} \sum_{i=1}^{n} \hat{\varepsilon}_{i}^{-2} z_{i} y_{i}$. However, this does not seem a good idea. Note that

$$
\hat{\beta}_{F n p M G M M}=\beta+\left[\hat{Q}_{z x}^{\prime} \hat{Q}_{z z}^{-1} \hat{Q}_{z x}\right]^{-1} \hat{Q}_{z x}^{\prime} \hat{Q}_{z z}^{-1}\left(n^{-1} \Sigma_{i} \hat{\varepsilon}_{i}^{-2} z_{i} \varepsilon_{i}\right) .
$$

Since the residuals $\hat{\varepsilon}_{i}$ will converge to the disturbances $\varepsilon_{i}$, we have plim $n^{-1} \Sigma_{i} \hat{\varepsilon}_{i}^{-2} z_{i} \varepsilon_{i}=$ plim $n^{-1} \Sigma_{i} \varepsilon_{i}^{-2} z_{i} \varepsilon_{i}=\operatorname{plim} n^{-1} \Sigma_{i} z_{i} / \varepsilon_{i}$, where the latter does not converge to a fixed value and hence not to zero, because $z_{i} / \varepsilon_{i}$ has no finite moments. Moreover, $\hat{Q}_{z z}$ and $\hat{Q}_{z x}$ are problematic too, because their probability limits differ from plim $n^{-1} Z^{\prime} \Sigma^{-1} Z$ and $\operatorname{plim} n^{-1} Z^{\prime} \Sigma^{-1} X$, because $E\left(1 / \varepsilon_{i}^{2}\right)$ does not exist either and thus differs from $1 / \sigma_{i}$. However, from our simulations it seems that nevertheless $\hat{\beta}_{F n p M G M M}$ is consistent and asymptotically normal, whereas $n\left[\hat{Q}_{z x}^{\prime} \hat{Q}_{z z}^{-1} \hat{Q}_{z x}\right]^{-1}$ is not consistent for $\operatorname{Var}\left(\hat{\beta}_{F n p M G M M}\right)$. These difficulties are similar to those regarding nonparametric feasible GLS ${ }^{7}$, for which $n^{-1} X^{\prime} \Sigma^{-1} X$ cannot be consistently estimated by $\hat{Q}_{x x}=n^{-1} \Sigma_{i} \hat{\varepsilon}_{i}^{-2} x_{i} x_{i}^{\prime}$. Newey (1993) discusses how the assessment of asymptotically optimal instruments $\Omega^{-1} Z$ should be approached in a nonparametric context.

In the simulations to follow, we will focus on the relatively simple case of heteroskedasticity in cross-sections. Typical time-series applications, where $\Omega$ may be nondiagonal and where the non-endogenous regressors and the instruments may not be strictly exogenous but just predetermined, require a more subtle approach. First, we examine standard GMM and its modification MGMM for the unrealistic situation that $\Omega$ is supposed to be known. Exploiting the true value of $\Omega$ will disclose what the potential differences will be between the for practitioners more interesting feasible implementations. Next, we examine what the actual losses are in terms of RMSE (root mean squared error) when $\Omega$ is assessed in the particular parametric or nonparametric implementations described above. We will also make comparisons with IV, and with the under simultaneity inconsistent estimators OLS, WLS (the special form of GLS when $\Omega$ is diagonal), FpWLS (given by $\left(X^{\prime} \hat{\Sigma}^{-1} X\right)^{-1} X^{\prime} \hat{\Sigma}^{-1} y$ ) and FnpWLS (given by $\hat{Q}_{x x}^{-1} \hat{Q}_{x y}$ ).

In addition to comparing bias, actual standard deviation, and root mean squared error, we will examine also how well the various estimators are able to assess their actual efficiency, by examining the bias in the estimates provided by the standard expressions for their standard errors. These calculations will be based on square roots of the diagonal elements of the following list of variance estimators ${ }^{8}$ :

\footnotetext{
${ }^{7}$ See ET Exercise 04.1.2 posed by Wansbeek (2004).

${ }^{8}$ As indicated above, the two variance estimates of this list involving $\hat{Q}$-type assessments are pretty naive. Also, $\widehat{\operatorname{Var}}\left(\hat{\beta}_{I V}\right)$ is improper in case $\Omega \neq I$, but a parametric and a nonparametric robustification for this variance are provided by $\widehat{\operatorname{Var}}_{p}\left(\hat{\beta}_{I V}\right)$ and $\widehat{\operatorname{Var}}_{n p}\left(\hat{\beta}_{I V}\right)$.
} 


$$
\begin{aligned}
& \widehat{\operatorname{Var}}\left(\hat{\beta}_{G M M}\right)=\hat{\sigma}_{\varepsilon}^{2}\left[X^{\prime} Z\left(Z^{\prime} \Omega Z\right)^{-1} Z^{\prime} X\right]^{-1}, \hat{\sigma}_{\varepsilon}^{2}=\left(y-X \hat{\beta}_{G M M}\right)^{\prime}\left(y-X \hat{\beta}_{G M M}\right) / n \\
& \widehat{\operatorname{Var}}\left(\hat{\beta}_{F p G M M}\right)=\left[X^{\prime} Z\left(Z^{\prime} \hat{\Sigma} Z\right)^{-1} Z^{\prime} X\right]^{-1}, \hat{\sigma}_{i}=h\left(z_{i}^{\prime} \hat{\gamma}\right) \\
& \widehat{\operatorname{Var}}\left(\hat{\beta}_{F n p G M M}\right)=n\left[X^{\prime} Z \hat{S}_{z z}^{-1} Z^{\prime} X\right]^{-1} \\
& \widehat{\operatorname{Var}}\left(\hat{\beta}_{M G M M}\right)=\hat{\sigma}_{\varepsilon}^{2}\left[X^{* \prime} P_{Z^{*}} X^{*}\right]^{-1}, \hat{\sigma}_{\varepsilon}^{2}=\left(y^{*}-X^{*} \hat{\beta}_{M G M M}\right)^{\prime}\left(y^{*}-X^{*} \hat{\beta}_{M G M M}\right) / n \\
& \widehat{\operatorname{Var}}\left(\hat{\beta}_{F p M G M M}\right)=\left[X^{\prime} \hat{\Sigma}^{-1} Z\left(Z^{\prime} \hat{\Sigma}^{-1} Z\right)^{-1} Z^{\prime} \hat{\Sigma}^{-1} X\right]^{-1}, \hat{\sigma}_{i}=h\left(z_{i}^{\prime} \hat{\gamma}\right) \\
& \widehat{\operatorname{Var}}\left(\hat{\beta}_{F n p M G M M}\right)=n^{-1}\left[\hat{Q}_{z x}^{\prime} \hat{Q}_{z z}^{-1} \hat{Q}_{z x}\right]^{-1} \\
& \widehat{\operatorname{Var}}\left(\hat{\beta}_{I V}\right)=\hat{\sigma}_{\varepsilon}^{2}\left[X^{\prime} P_{Z} X\right]^{-1}, \hat{\sigma}_{\varepsilon}^{2}=\left(y-X \hat{\beta}_{I V}\right)^{\prime}\left(y-X \hat{\beta}_{I V}\right) / n \\
& \widehat{\operatorname{Var}}\left(\hat{\beta}_{I V}\right)=\left[X^{\prime} P_{Z} X\right]^{-1} X^{\prime} P_{Z} \hat{\Sigma} P_{Z} X\left[X^{\prime} P_{Z} X\right]^{-1}, \hat{\sigma}_{i}=h\left(z_{i}^{\prime} \hat{\gamma}\right) \\
& \widehat{\operatorname{Var}}_{n p}\left(\hat{\beta}_{I V}\right)=n\left[X^{\prime} P_{Z} X\right]^{-1} X^{\prime} Z\left(Z^{\prime} Z\right)^{-1} \hat{S}_{z z}\left(Z^{\prime} Z\right)^{-1} Z^{\prime} X\left[X^{\prime} P_{Z} X\right]^{-1} \\
& \widehat{\operatorname{Var}}^{-1}\left(\hat{\beta}_{F n p W L S}\right)=n^{-1} \hat{Q}_{x x}^{-1}, \hat{Q}_{x x}=n^{-1} \sum_{\iota=1}^{n} \hat{\varepsilon}_{i}^{-2} x_{i} x_{i}^{\prime}, \hat{\varepsilon}_{i}=y_{i}-x_{i}^{\prime} \hat{\beta}_{I V}
\end{aligned}
$$

Of course, for accurate inference in the form of tests or confidence intervals further aspects are relevant as well, but a relatively small bias in both the estimates of coefficients and in their estimated standard deviations (standard errors) seem of primary concern, on top of the actual relative magnitude of the RMSE.

\section{Simulation design for a heteroskedastic cross-section model}

We shall design a data generating process (DGP) in which we can easily change the seriousness and characteristics of the heteroskedasticity, the degree of simultaneity, the strength of the instruments, the significance of individual regressors and the general fit of the relationship. To assure that the first two moments of IV estimators exist we choose the degree of overidentification to be 2. In the DGP we allow for the presence of an intercept, another exogenous regressor and one possibly endogenous regressor, hence $K_{1}=2, K_{2}=1$ and $K=3$. The two exogenous regressors, which are also used as instruments $\left(L_{1}=K_{1}\right)$, are $x_{i 1}=1$ and $x_{i 2} \sim i i d N(0,1)$; the three external instruments $\left(L_{2}=3\right)$ are generated too as mutually independent $z_{i j} \sim \operatorname{iidN}(0,1)$ for $j=3,4,5 ; i=1, \ldots, n$. Of course, the two endogenous variables $x_{i 3}$ and $y_{i}$, and the pattern of the heteroskedasticity $\left(\omega_{1}, \ldots, \omega_{n}\right)$ in the disturbances $\varepsilon_{i}$, where the $\omega_{i}$ are the diagonal elements of $\Omega$, have to be designed such that these seem very realistic for typical cross-section applications.

The structural form equation will be generated as

$$
y_{i}=\beta_{1}+\beta_{2} x_{i 2}+\beta_{3} x_{i 3}+\varepsilon_{i}
$$

and the reduced form equation for $x_{i 3}$ by

$$
x_{i 3}=\pi_{31}+\pi_{32} x_{i 2}+\pi_{33} z_{i 3}+\pi_{34} z_{i 4}+\pi_{35} z_{i 5}+v_{i},
$$

where

$$
\begin{aligned}
v_{i} & =\sigma_{v} \omega_{i}^{1 / 2} v_{i}^{\circ}, \text { with } v_{i}^{\circ} \sim \operatorname{iidN}(0,1), \\
\varepsilon_{i} & =\sigma_{\varepsilon} \omega_{i}^{1 / 2}\left[\rho v_{i}^{\circ}+\left(1-\rho^{2}\right)^{1 / 2} \varepsilon_{i}^{\circ}\right], \text { with } \varepsilon_{i}^{\circ} \sim \operatorname{iidN}(0,1) .
\end{aligned}
$$

Hence, the reduced form disturbances $v_{i} \sim N\left(0, \sigma_{v}^{2} \omega_{i}\right)$ and the structural equation disturbances $\varepsilon_{i} \sim N\left(0, \sigma_{\varepsilon}^{2} \omega_{i}\right)$ are affected by the same heteroskedasticity pattern. Parameter $\rho \in(-1,+1)$ is the correlation coefficient of $\varepsilon_{i}$ and $v_{i}$ and expresses the degree of simultaneity. 
Using $n^{-1} \sum_{i=1}^{n} E\left(v_{i}^{2}\right)=\sigma_{v}^{2} n^{-1} \sum_{i=1}^{n} \omega_{i}=\sigma_{v}^{2}$, the joint strength of the three external instruments is determined by the scaled concentration parameter inspired scalar quantity

$$
\mu^{2}=\frac{n}{3} \frac{\pi_{33}^{2}+\pi_{34}^{2}+\pi_{35}^{2}}{\sigma_{v}^{2}}
$$

which implies $\operatorname{Var}\left(x_{i 3}\right)=\pi_{32}^{2}+\sigma_{v}^{2}\left(3 \mu^{2} / n+\omega_{i}\right)$. All the external instruments and exogenous regressor $x_{i 2}$ have normalized variance unity. Thus, treating endogenous regressor $x_{i 3}$ similarly requires $\pi_{32}^{2}+\sigma_{v}^{2}\left(3 \mu^{2} / n+1\right)=1$. Therefore we will choose

$$
\sigma_{v}^{2}=\frac{1-\pi_{32}^{2}}{3 \mu^{2} / n+1}
$$

Note that $\pi_{32}^{2}<1$, since $\pi_{32}$ is the correlation coefficient determining the multicollinearity between the structural form regressors $x_{i 2}$ and $x_{i 3}$. To have all three external instruments equally weak or strong, we should take

$$
\pi_{33}=\pi_{34}=\pi_{35}=\left|\mu \sigma_{v}\right| / \sqrt{n} .
$$

The heteroskedasticity pattern will follow a so-called multiplicative form determined by the $\operatorname{iidN}(0,1)$ series $x_{i 2}$ and $z_{i 3}$ (hence by one internal and one external instrument), but could be driven by another in practice unobserved independent variable $\eta_{i}^{\circ} \sim \operatorname{iidN}(0,1)$ as well. A parameter $\phi \geq 0$ determines the seriousness of the heteroskedasticity, where $\phi=0$ implies homoskedasticity. When $\phi>0$ a parameter $\lambda$, with $0 \leq \lambda \leq 1$, determines the relative importance of the observed variables $\left(x_{i 2}\right.$ and $\left.z_{i 3}\right)$ and the unobserved variable $\left(\eta_{i}^{\circ}\right)$ regarding the heteroskedasticity. And, if $\lambda>0$, a parameter $\kappa$, with $0 \leq \kappa \leq 1$, determines the relative importance of $x_{i 2}$ and $z_{i 3}$ regarding any heteroskedasticity. This is achieved by generating the variable

$$
g_{i}=-\phi^{2} / 2+\phi\left\{\lambda^{1 / 2}\left[\kappa^{1 / 2} x_{i 2}+(1-\kappa)^{1 / 2} z_{i 3}\right]+(1-\lambda)^{1 / 2} \eta_{i}^{\circ}\right\} \sim i i d N\left(-\phi^{2} / 2, \phi^{2}\right),
$$

and taking

$$
\omega_{i}=\exp \left(g_{i}\right)
$$

which follows a lognormal distribution with $E\left(\omega_{i}\right)=1$ and $\operatorname{Var}\left(\omega_{i}\right)=\exp \left(\phi^{2}\right)-1$. Since about $99 \%$ of the drawings $g_{i}$ will be in the interval $\left[-\phi^{2} / 2-2.58 \phi,-\phi^{2} / 2+2.58 \phi\right]$, also $99 \%$ of the drawings $\omega_{i}$ will fall in the interval

$$
\left[\exp \left(-\phi^{2} / 2-2.58 \phi\right), \exp \left(-\phi^{2} / 2+2.58 \phi\right)\right]
$$

Table 4.1 presents the bounds of these intervals, both for $\omega_{i}$ and for $\omega_{i}^{1 / 2}$, for particular values of $\phi$. From these we learn that $\phi \geq 1$ implies pretty serious heteroskedasticity, whereas we may qualify it mild when $\phi<0.3$, say. 


\begin{tabular}{|c|c|c|c|c|}
\hline Table 4.1 & \multirow{2}{*}{\multicolumn{4}{|c|}{$\begin{array}{c}\text { Heteroskedasticity for different values of } \phi \\
\text { bounds of } 99 \% \text { intervals }\end{array}$}} \\
\hline \multirow[t]{2}{*}{$\phi$} & & & & \\
\hline & \multicolumn{2}{|c|}{$\omega_{i}^{1 / 2}$} & \multicolumn{2}{|c|}{$\omega_{i}$} \\
\hline 0.2 & 0.76 & 1.28 & 0.59 & 1.64 \\
\hline 0.4 & 0.57 & 1.61 & 0.33 & 2.59 \\
\hline 0.6 & 0.42 & 1.98 & 0.18 & 3.93 \\
\hline 0.8 & 0.30 & 2.39 & 0.09 & 5.72 \\
\hline 1.0 & 0.21 & 2.83 & 0.05 & 8.00 \\
\hline 1.2 & 0.15 & 3.28 & 0.02 & 10.76 \\
\hline 1.4 & 0.10 & 3.73 & 0.01 & 13.90 \\
\hline 1.6 & 0.07 & 4.15 & 0.00 & 17.25 \\
\hline
\end{tabular}

The design defined above, with the deliberate choices (4.6) and (4.7), has yet, apart from the sample size $n, 11$ free parameters, namely: $\beta_{1}, \beta_{2}, \beta_{3}$ and $\sigma_{\varepsilon} ; \rho, \pi_{31}, \pi_{32}$ and $\mu^{2}$; and $\phi, \lambda$ and $\kappa$. For all the estimation techniques to be examined their estimation errors are invariant with respect to $\beta$. For instance, $\hat{\beta}_{O L S}-\beta=\left(X^{\prime} X\right)^{-1} X^{\prime} \varepsilon$ and $\hat{\beta}_{I V}-\beta=$ $\left(X^{\prime} P_{Z} X\right)^{-1} X^{\prime} P_{Z} \varepsilon$, etc. Therefore, the bias, variance and mean squared errors of the coefficient estimates will for all techniques be invariant with respect to $\beta$ too. So, from that point of view we may choose any values for the structural coefficients. However, since the estimation errors do depend on $\varepsilon$ and hence on $\sigma_{\varepsilon}$, by imposing the habitual normalization $\sigma_{\varepsilon}=1$, the magnitude of the estimation errors will be affected and thus the findings for the bias, variance and mean squared errors too. This is of less concern, though, when we focus on the relative magnitude of the estimation errors for the different techniques to be examined. Moreover, we can choose values for $\beta_{2}$ and $\beta_{3}$ such that, in combination with $\sigma_{\varepsilon}=1$, values (averaged over all replications) for $t$ and $F$ tests or for $R^{2}$ are found which are not uncommon in cross-section regression analysis. A loosely defined population coefficient of determination for simultaneous heteroskedastic model (4.1) is given by

$$
R_{p}^{2}=1-\frac{\sum_{i=1}^{n} \operatorname{Var}\left(\varepsilon_{i}\right)}{\sum_{i=1}^{n}\left[\operatorname{Var}\left(\beta_{2} x_{i 2}+\beta_{3} x_{i 3}\right)+\operatorname{Var}\left(\varepsilon_{i}\right)\right]}=1-\frac{1}{\beta_{2}^{2}+2 \beta_{2} \beta_{3} \pi_{32}+\beta_{3}^{2}+1} .
$$

Taking equal values for the two slope coefficients then yields

$$
\beta_{2}^{2}=\beta_{3}^{2}=R_{p}^{2} /\left[2\left(1-R_{p}^{2}\right)\left(1+\pi_{32}\right)\right] .
$$

For $0.1 \leq R_{p}^{2} \leq 0.2$ and $0 \leq \pi_{32} \leq 0.8$ this implies positive solutions in the range $0.18 \leq \beta_{2}=\beta_{3} \leq 0.35$. What we will do is simply take $\beta_{2}=\beta_{3}=0.25$ and monitor averages of statistics over all the replications of the Monte Carlo which monitor the practical relevance of the cases examined. These cases take particular combinations from the grid given in Table 4.2. Choosing $\beta_{1}=0$ and $\pi_{31}=0$, but incorporating the intercept always in the model and in the matrix of instruments, will not affect the findings regarding inference on $\beta_{2}$ and $\beta_{3}$.

\begin{tabular}{llll}
\hline Table $\mathbf{4 . 2}$ & Grid of design parameter values in the simulation \\
\hline$n=\{50,200\}$ & & & \\
$\beta_{1}=0$ & $\beta_{2}=0.25$ & $\beta_{3}=0.25$ & $\sigma_{\varepsilon}=1$ \\
$\rho=\{0.1,0.5\}$ & $\pi_{31}=0$ & $\pi_{32}=\{0,0.8\}$ & $\mu^{2}=\{2,10,50\}$ \\
$\phi=\{0.5,1\}$ & $\lambda=\{0,0.2,0.5,1\}$ & $\kappa=\{0.2,0.5,0.8\}$ & \\
\hline
\end{tabular}


In the tables in the next section with results on the various estimators we limit the analysis to just 12 different cases as defined in Table 4.3. This table also presents the value of $\sigma_{v}$ that results according to (4.6). The final two columns contain the average (with standard deviation between parentheses) over all replications of the standard OLS $R^{2}$ statistic (thus neglecting the simultaneity and any heteroskedasticity) in the structural model (4.1) and of the $F_{3, n-L}$ test statistic on the joint significance of the external instruments in the reduced form equation (4.2) when estimated by OLS (thus again neglecting any heteroskedasticity). Both measures have their drawbacks, but they are only used here to give a rough impression of major characteristics of the DGPs, namely their fit and the strength of the external instruments. Note that there is a reasonable correspondence between the values of $\mu^{2}$ and the average $F_{3, n-L}$ statistic. Due to the inconsistency of OLS results in the structural model, only in case E (where the simultaneity is very mild) the average $R^{2}$ statistic is in the range of the aimed at $R_{p}^{2}$ value. In the next section further evidence will be discussed regarding the empirical relevance of the chosen designs.

\begin{tabular}{ccccccccccc}
\hline Table 4.3 & \multicolumn{8}{c}{ Examined cases of design parameter value combinations, and results } \\
& on $\sigma_{v}, R^{2}$ and $F_{3, n-L}\left(\beta_{1}=0, \beta_{2}=\beta_{3}=0.25, \sigma_{\varepsilon}=1, \pi_{31}=0\right)$ \\
\hline Case & $n$ & $\rho$ & $\pi_{32}$ & $\mu^{2}$ & $\phi$ & $\lambda$ & $\kappa$ & $\sigma_{v}$ & $R^{2}$ & $F_{3, n-L}$ \\
\hline A & 200 & 0.5 & 0 & 50 & 1 & 1 & 0.5 & 0.76 & $0.35(0.07)$ & $52.56(13.36)$ \\
B & 200 & 0.5 & 0 & 10 & 1 & 1 & 0.5 & 0.93 & $0.43(0.08)$ & $11.47(4.61)$ \\
C & 200 & 0.5 & 0 & 2 & 1 & 1 & 0.5 & 0.99 & $0.45(0.08)$ & $3.24(2.10)$ \\
D & 200 & 0.5 & 0 & 10 & 0.5 & 1 & 0.5 & 0.93 & $0.43(0.06)$ & $11.16(4.21)$ \\
E & 200 & 0.1 & 0 & 10 & 1 & 1 & 0.5 & 0.93 & $0.17(0.07)$ & $11.47(4.61)$ \\
F & 200 & 0.5 & 0.8 & 10 & 1 & 1 & 0.5 & 0.56 & $0.43(0.07)$ & $11.47(4.61)$ \\
G & 200 & 0.5 & 0 & 10 & 1 & 1 & 0.2 & 0.93 & $0.43(0.08)$ & $11.58(4.77)$ \\
H & 200 & 0.5 & 0 & 10 & 1 & 1 & 0.8 & 0.93 & $0.43(0.08)$ & $11.35(4.44)$ \\
I & 50 & 0.5 & 0 & 10 & 1 & 1 & 0.5 & 0.79 & $0.39(0.14)$ & $11.92(6.01)$ \\
J & 200 & 0.5 & 0 & 10 & 1 & 0.5 & 0.5 & 0.93 & $0.43(0.07)$ & $11.27(4.35)$ \\
K & 200 & 0.5 & 0 & 10 & 1 & 0.2 & 0.5 & 0.93 & $0.43(0.06)$ & $11.16(4.21)$ \\
L & 200 & 0.5 & 0 & 10 & 1 & 0 & 0.5 & 0.93 & $0.43(0.05)$ & $11.07(4.11)$ \\
\hline
\end{tabular}

The simulation estimates are obtained from 10,000 Monte Carlo replications. In each replication new independent realizations have been drawn for $\varepsilon_{i}^{\circ}, x_{i 2}, z_{i 3}, z_{i 4}, z_{i 5}, v_{i}^{\circ}$ and $\eta_{i}^{\circ}$, so the Monte Carlo averages estimate unconditional moments. ${ }^{9}$

\section{Simulation results}

In Table 5.1 we collect results for the cases A, B and C. They all concern the larger sample size, have serious heteroskedasticity, substantial simultaneity, and no multicollinearity between $x_{i 2}$ and $x_{i 3}$. These three cases just differ in the strength of the three external instruments, as can be seen from Table 4.3.

\footnotetext{
${ }^{9}$ Advantages and disadvantages of conditioning or not on exogenous variables in simulation experiments are discussed in Kiviet (2012).
} 
Because the regressors $x_{2}$ and $x_{3}$ are uncorrelated it can easily be derived that even the inconsistent coefficient vector estimators (all the least-squares variants) yield a consistent estimator for element $\beta_{2}$, the coefficient of the exogenous regressor $x_{2}$. So, it should not surprise that all estimators produce almost unbiased results for $\beta_{2}$, also when instruments are weak. Regarding $\beta_{3}$ it are only the consistent estimators that have moderate bias, provided the instruments are not weak. However, also FnpWLS performs surprisingly well for non-weak instruments. For the weak instrument case $\mathrm{C}$ also the consistent estimators of $\beta_{3}$ show substantial bias, in particular IV and those based on standard GMM. The most remarkable result, however, is that the MGMM and FpMGMM estimators are substantially more efficient than standard GMM. In these three cases they reduce the RMSE by about 40 or $50 \%$, irrespective of the strength of the instruments, as can be seen easily from the rrmse (relative RMSE) columns which presents the RMSE divided by that of unfeasible standard GMM.

Further note that both the parametric and the nonparametric feasible implementations of standard GMM are very close to the unfeasible estimator. That the latter does not use optimal instruments is evident from the unfeasible MGMM results. Also the parametric feasible version of MGMM works well, but the nonparametric implementation does not improve on standard GMM. It is also noteworthy that we find that standard GMM is in fact not all that much better than IV, illustrating that the efficiency gain due to taking $\Omega$ into account is largely offset by the fact that the instruments used by the standard implementation of GMM are weaker than those used by IV. This weakening is prevented by MGMM through weighing the instruments by the same weights as used for the variables in the transformed model, inducing remarkable reductions both in bias and standard deviation. Another interesting finding from Table 5.1 is that on the basis of their RMSE the inconsistent estimators often outperform consistent GMM and occasionally even MGMM (just for $\beta_{2}$ ). This is in line with results reported on OLS and IV in Kiviet (2013): weakness of instruments is often more detrimental to estimator accuracy than invalidity of instruments. Therefore, when the instruments are weak, FpWLS is found to be substantially better than unfeasible GMM. Despite its bias due to its inconsistency, its standard deviation is smaller due to using strong (though invalid) instruments, whereas consistent GMM has nevertheless substantial bias and standard deviations, due to the weakness of its instruments.

Regarding the empirical relevance of the models examined in cases $\mathrm{A}, \mathrm{B}$ and $\mathrm{C}$ it is useful to examine the quantities $\beta_{2} / s d\left(\hat{\beta}_{2}\right)$ and $\beta_{3} / s d\left(\hat{\beta}_{3}\right)$ for the different techniques. This ratio has correspondences with the inverse of the coefficient of variation and with a stylized $t$-ratio. For case $\mathrm{A}$ it is found to be in the range $(2.12,5.43)$, for case $\mathrm{B}$ in the range $(1.19,5.68)$ and for $\mathrm{C}$ in $(0.45,6.25)$. Note that in case $\mathrm{C}$ the estimators based on external weak instruments have for the estimate of $\beta_{3}$ such a large standard deviation in comparison to the true coefficient value of 0.25 that they do not enable to produce very useful inference. And, although OLS and especially WLS have quite a reasonable dispersion here, they are nevertheless unfit for producing informative inference too, due to their huge bias, which is about twice the true value of the estimated coefficient. However, in case A values for the true standard deviation are found which, assuming that in practice these could be estimated accurately (to be examined at the end of this section), would enable to construct confidence intervals of a relative width not uncommon in practice. 


\begin{tabular}{|c|c|c|c|c|c|c|c|c|c|}
\hline \multicolumn{2}{|c|}{ Table 5.1} & \multicolumn{8}{|c|}{ Simulation results on coefficient estimates for cases A, B and C } \\
\hline \multirow[b]{2}{*}{ Case } & & \multicolumn{4}{|l|}{$\beta_{2}$} & \multicolumn{4}{|l|}{$\beta_{3}$} \\
\hline & & bias & st.dv & rmse & rrmse & bias & st.dv & rmse & rrmse \\
\hline \multirow[t]{11}{*}{ A: } & GMM & 0.002 & 0.081 & 0.081 & 1.000 & 0.005 & 0.115 & 0.115 & 1.000 \\
\hline & FpGMM & 0.002 & 0.082 & 0.082 & 1.005 & 0.005 & 0.115 & 0.115 & 1.003 \\
\hline & FnpGMM & 0.002 & 0.081 & 0.081 & 0.998 & 0.005 & 0.114 & 0.114 & 0.999 \\
\hline & MGMM & 0.001 & 0.045 & 0.045 & 0.551 & 0.002 & 0.068 & 0.068 & 0.590 \\
\hline & FpMGMM & 0.001 & 0.047 & 0.047 & 0.572 & 0.002 & 0.070 & 0.070 & 0.610 \\
\hline & FnpMGMM & 0.001 & 0.085 & 0.085 & 1.040 & 0.005 & 0.117 & 0.117 & 1.021 \\
\hline & IV & 0.001 & 0.086 & 0.086 & 1.051 & 0.005 & 0.118 & 0.118 & 1.027 \\
\hline & OLS & 0.001 & 0.078 & 0.078 & 0.958 & 0.375 & 0.091 & 0.386 & 3.366 \\
\hline & WLS & 0.003 & 0.046 & 0.046 & 0.565 & 0.225 & 0.058 & 0.232 & 2.028 \\
\hline & FpWLS & 0.003 & 0.047 & 0.047 & 0.581 & 0.228 & 0.062 & 0.237 & 2.064 \\
\hline & FnpWLS & 0.001 & 0.085 & 0.085 & 1.038 & 0.010 & 0.116 & 0.116 & 1.015 \\
\hline \multirow[t]{11}{*}{ B: } & GMM & 0.002 & 0.082 & 0.082 & 1.000 & 0.020 & 0.211 & 0.212 & 1.000 \\
\hline & FpGMM & 0.002 & 0.082 & 0.083 & 1.005 & 0.020 & 0.211 & 0.212 & 1.003 \\
\hline & FnpGMM & 0.002 & 0.082 & 0.082 & 0.999 & 0.020 & 0.211 & 0.212 & 1.001 \\
\hline & MGMM & 0.001 & 0.045 & 0.045 & 0.547 & 0.007 & 0.123 & 0.123 & 0.582 \\
\hline & FpMGMM & 0.001 & 0.047 & 0.047 & 0.568 & 0.008 & 0.127 & 0.127 & 0.602 \\
\hline & FnpMGMM & -0.000 & 0.085 & 0.085 & 1.037 & 0.022 & 0.214 & 0.215 & 1.017 \\
\hline & IV & -0.000 & 0.086 & 0.086 & 1.048 & 0.022 & 0.215 & 0.216 & 1.022 \\
\hline & OLS & 0.001 & 0.075 & 0.075 & 0.912 & 0.465 & 0.095 & 0.474 & 2.243 \\
\hline & WLS & 0.003 & 0.044 & 0.044 & 0.532 & 0.386 & 0.062 & 0.390 & 1.846 \\
\hline & FpWLS & 0.003 & 0.045 & 0.045 & 0.546 & 0.385 & 0.064 & 0.390 & 1.845 \\
\hline & FnpWLS & 0.000 & 0.085 & 0.085 & 1.032 & 0.030 & 0.209 & 0.212 & 1.000 \\
\hline \multirow[t]{11}{*}{$\mathrm{C}:$} & GMM & 0.005 & 0.089 & 0.089 & 1.000 & 0.117 & 0.528 & 0.541 & 1.000 \\
\hline & FpGMM & 0.004 & 0.089 & 0.089 & 1.005 & 0.117 & 0.531 & 0.544 & 1.006 \\
\hline & FnpGMM & 0.004 & 0.091 & 0.091 & 1.018 & 0.118 & 0.553 & 0.566 & 1.046 \\
\hline & MGMM & 0.001 & 0.046 & 0.046 & 0.512 & 0.037 & 0.268 & 0.271 & 0.500 \\
\hline & FpMGMM & 0.001 & 0.047 & 0.047 & 0.529 & 0.040 & 0.276 & 0.279 & 0.516 \\
\hline & FnpMGMM & 0.001 & 0.091 & 0.091 & 1.025 & 0.124 & 0.529 & 0.543 & 1.004 \\
\hline & IV & 0.001 & 0.091 & 0.091 & 1.028 & 0.124 & 0.528 & 0.542 & 1.002 \\
\hline & OLS & 0.001 & 0.074 & 0.074 & 0.829 & 0.492 & 0.097 & 0.502 & 0.928 \\
\hline & WLS & 0.002 & 0.040 & 0.040 & 0.454 & 0.471 & 0.062 & 0.475 & 0.878 \\
\hline & FpWLS & 0.002 & 0.042 & 0.042 & 0.470 & 0.468 & 0.064 & 0.473 & 0.874 \\
\hline & FnpWLS & 0.001 & 0.087 & 0.087 & 0.973 & 0.133 & 0.442 & 0.461 & 0.853 \\
\hline
\end{tabular}

In all the further cases to be examined, we shall keep $\mu^{2}=10$, so the instruments are not very weak, but certainly not strong. Thus, from now on, case B should be considered the reference case. Case D in Table 5.2 is similar to case B, apart from the seriousness of the heteroskedasticity. We see that $\phi=0.5$ leads to similar though more moderate relative differences, with efficiency gains by MGMM still around $15 \%$. All remaining cases have $\phi=1$ again. Table 5.2 also contains cases $\mathrm{E}$ and $\mathrm{F}$. In case E the simultaneity is mild. This is seen to have no effects on the relative performance of MGMM, but now the inconsistent estimators have minor bias for both coefficients and therefore they have better RMSE (except FnpWLS). Case F differs from B just regarding 
the occurrence of substantial multicollinearity between $x_{i 2}$ and $x_{i 3}$, which reduces the value of $\sigma_{v}$. Note that the rrmse results on $\beta_{3}$ are invariant regarding $\pi_{23}$, but not those for $\beta_{2}$. The least-squares based estimators for $\beta_{2}$ are now inconsistent too. All standard deviations are much higher and the bias in estimates for $\beta_{3}$ infects the estimates of $\beta_{2}$ with bias. As before, MGMM and FpMGMM perform best, whereas again FnpWLS remarkably keeps pace with FnpGMM.

\begin{tabular}{|c|c|c|c|c|c|c|c|c|c|}
\hline \multicolumn{2}{|c|}{ Table 5.2} & \multicolumn{8}{|c|}{ Simulation results on coefficient estimates for cases $\mathrm{D}, \mathrm{E}$ and $\mathrm{F}$} \\
\hline \multirow[b]{2}{*}{ Case } & & \multicolumn{4}{|l|}{$\beta_{2}$} & \multicolumn{4}{|l|}{$\beta_{3}$} \\
\hline & & bias & st.dv & rmse & rrmse & bias & st.dv & rmse & rrmse \\
\hline \multirow[t]{11}{*}{ D: } & GMM & 0.002 & 0.075 & 0.075 & 1.000 & 0.019 & 0.204 & 0.205 & 1.000 \\
\hline & FpGMM & 0.002 & 0.075 & 0.075 & 1.001 & 0.019 & 0.204 & 0.205 & 1.000 \\
\hline & FnpGMM & 0.001 & 0.075 & 0.075 & 1.007 & 0.019 & 0.206 & 0.207 & 1.009 \\
\hline & MGMM & 0.001 & 0.063 & 0.063 & 0.844 & 0.014 & 0.176 & 0.177 & 0.863 \\
\hline & FpMGMM & 0.001 & 0.065 & 0.065 & 0.872 & 0.015 & 0.182 & 0.182 & 0.888 \\
\hline & FnpMGMM & 0.001 & 0.076 & 0.076 & 1.011 & 0.019 & 0.206 & 0.207 & 1.007 \\
\hline & IV & 0.001 & 0.075 & 0.075 & 1.006 & 0.019 & 0.205 & 0.206 & 1.003 \\
\hline & OLS & 0.001 & 0.066 & 0.066 & 0.883 & 0.466 & 0.070 & 0.471 & 2.297 \\
\hline & WLS & 0.001 & 0.057 & 0.057 & 0.757 & 0.450 & 0.063 & 0.455 & 2.217 \\
\hline & FpWLS & 0.001 & 0.059 & 0.059 & 0.783 & 0.444 & 0.064 & 0.449 & 2.188 \\
\hline & FnpWLS & 0.001 & 0.075 & 0.075 & 1.003 & 0.028 & 0.200 & 0.202 & 0.983 \\
\hline \multirow[t]{11}{*}{$\mathrm{E}:$} & GMM & 0.001 & 0.083 & 0.083 & 1.000 & 0.003 & 0.211 & 0.211 & 1.000 \\
\hline & FpGMM & 0.001 & 0.083 & 0.083 & 1.007 & 0.003 & 0.211 & 0.211 & 1.003 \\
\hline & FnpGMM & 0.001 & 0.083 & 0.083 & 1.000 & 0.002 & 0.211 & 0.211 & 1.000 \\
\hline & MGMM & 0.001 & 0.045 & 0.045 & 0.547 & 0.002 & 0.124 & 0.124 & 0.589 \\
\hline & FpMGMM & 0.001 & 0.047 & 0.047 & 0.568 & 0.002 & 0.128 & 0.128 & 0.607 \\
\hline & FnpMGMM & 0.000 & 0.086 & 0.086 & 1.040 & 0.003 & 0.215 & 0.215 & 1.020 \\
\hline & IV & 0.000 & 0.087 & 0.087 & 1.051 & 0.003 & 0.216 & 0.216 & 1.023 \\
\hline & OLS & 0.001 & 0.085 & 0.085 & 1.021 & 0.093 & 0.108 & 0.143 & 0.677 \\
\hline & WLS & 0.001 & 0.045 & 0.045 & 0.541 & 0.078 & 0.065 & 0.101 & 0.479 \\
\hline & FpWLS & 0.001 & 0.047 & 0.047 & 0.562 & 0.077 & 0.067 & 0.102 & 0.485 \\
\hline & FnpWLS & 0.000 & 0.086 & 0.086 & 1.038 & 0.004 & 0.212 & 0.212 & 1.007 \\
\hline \multirow[t]{11}{*}{$\mathrm{F}:$} & GMM & -0.024 & 0.280 & 0.281 & 1.000 & 0.034 & 0.351 & 0.353 & 1.000 \\
\hline & FpGMM & -0.025 & 0.281 & 0.282 & 1.002 & 0.034 & 0.352 & 0.354 & 1.003 \\
\hline & FnpGMM & -0.024 & 0.280 & 0.281 & 0.999 & 0.033 & 0.351 & 0.353 & 1.001 \\
\hline & MGMM & -0.009 & 0.169 & 0.170 & 0.603 & 0.012 & 0.205 & 0.205 & 0.582 \\
\hline & FpMGMM & -0.010 & 0.175 & 0.176 & 0.624 & 0.013 & 0.212 & 0.212 & 0.602 \\
\hline & FnpMGMM & -0.029 & 0.282 & 0.284 & 1.009 & 0.037 & 0.357 & 0.358 & 1.017 \\
\hline & IV & -0.029 & 0.284 & 0.285 & 1.014 & 0.036 & 0.359 & 0.360 & 1.022 \\
\hline & OLS & -0.619 & 0.145 & 0.636 & 2.259 & 0.775 & 0.159 & 0.791 & 2.243 \\
\hline & WLS & -0.511 & 0.092 & 0.520 & 1.847 & 0.643 & 0.103 & 0.651 & 1.846 \\
\hline & FpWLS & -0.510 & 0.095 & 0.519 & 1.845 & 0.641 & 0.107 & 0.650 & 1.845 \\
\hline & FnpWLS & -0.039 & 0.277 & 0.279 & 0.993 & 0.049 & 0.349 & 0.353 & 1.000 \\
\hline
\end{tabular}

Cases $\mathrm{G}$ and $\mathrm{H}$ in Table 5.3 differ from B only in $\kappa$, so in whether either $x_{i 2}$ or $z_{i 3}$ is 
the major source of the heteroskedasticity. The effects of $\kappa$ are found to be moderate and both cases again show spectacular efficiency gains by (feasible parametric) MGMM. The only difference between cases I and B is the smaller sample size. This clearly mitigates the gains by MGMM over standard GMM, but they are still around 30\%. We also note that dividing the sample size by a factor 4 doubles about the standard deviation of the estimators of the coefficient of the exogenous regressor when it is uncorrelated with the endogenous regressor, but the effect on the standard deviation of the coefficient estimate regarding the endogenous regressor is less mechanic for most techniques.

\begin{tabular}{|c|c|c|c|c|c|c|c|c|c|}
\hline \multicolumn{2}{|c|}{ Table 5.3} & \multicolumn{8}{|c|}{ Simulation results on coefficient estimates for cases $\mathrm{G}, \mathrm{H}$ and I } \\
\hline \multirow[b]{2}{*}{ Case } & & \multicolumn{4}{|c|}{$\beta_{2}$} & \multicolumn{4}{|l|}{$\beta_{3}$} \\
\hline & & bias & st.dv & rmse & rrmse & bias & st.dv & rmse & rrmse \\
\hline \multirow[t]{11}{*}{ G: } & GMM & 0.002 & 0.075 & 0.075 & 1.000 & 0.021 & 0.215 & 0.216 & 1.000 \\
\hline & FpGMM & 0.002 & 0.075 & 0.075 & 1.002 & 0.021 & 0.216 & 0.217 & 1.004 \\
\hline & FnpGMM & 0.002 & 0.075 & 0.075 & 0.998 & 0.021 & 0.215 & 0.216 & 1.001 \\
\hline & MGMM & 0.001 & 0.044 & 0.044 & 0.594 & 0.007 & 0.123 & 0.123 & 0.571 \\
\hline & FpMGMM & 0.001 & 0.046 & 0.046 & 0.613 & 0.008 & 0.127 & 0.127 & 0.588 \\
\hline & FnpMGMM & 0.000 & 0.077 & 0.077 & 1.027 & 0.024 & 0.222 & 0.223 & 1.033 \\
\hline & IV & 0.000 & 0.077 & 0.077 & 1.035 & 0.024 & 0.223 & 0.224 & 1.039 \\
\hline & OLS & 0.001 & 0.068 & 0.068 & 0.904 & 0.465 & 0.096 & 0.475 & 2.199 \\
\hline & WLS & 0.002 & 0.043 & 0.043 & 0.572 & 0.386 & 0.063 & 0.391 & 1.810 \\
\hline & FpWLS & 0.002 & 0.044 & 0.044 & 0.586 & 0.385 & 0.065 & 0.390 & 1.807 \\
\hline & FnpWLS & 0.000 & 0.076 & 0.076 & 1.021 & 0.031 & 0.217 & 0.220 & 1.018 \\
\hline \multirow[t]{11}{*}{$\mathrm{H}:$} & GMM & 0.002 & 0.091 & 0.091 & 1.000 & 0.019 & 0.204 & 0.205 & 1.000 \\
\hline & FpGMM & 0.002 & 0.091 & 0.091 & 1.006 & 0.019 & 0.205 & 0.205 & 1.002 \\
\hline & FnpGMM & 0.002 & 0.091 & 0.091 & 0.998 & 0.019 & 0.204 & 0.205 & 1.001 \\
\hline & MGMM & 0.001 & 0.045 & 0.045 & 0.500 & 0.007 & 0.123 & 0.123 & 0.601 \\
\hline & FpMGMM & 0.001 & 0.047 & 0.047 & 0.522 & 0.008 & 0.127 & 0.127 & 0.621 \\
\hline & FnpMGMM & 0.000 & 0.093 & 0.093 & 1.023 & 0.020 & 0.206 & 0.207 & 1.009 \\
\hline & IV & 0.000 & 0.094 & 0.094 & 1.036 & 0.020 & 0.206 & 0.207 & 1.010 \\
\hline & OLS & 0.001 & 0.081 & 0.081 & 0.897 & 0.465 & 0.095 & 0.474 & 2.315 \\
\hline & WLS & 0.002 & 0.044 & 0.044 & 0.489 & 0.385 & 0.061 & 0.390 & 1.903 \\
\hline & FpWLS & 0.002 & 0.046 & 0.046 & 0.504 & 0.385 & 0.064 & 0.390 & 1.903 \\
\hline & FnpWLS & 0.000 & 0.092 & 0.092 & 1.017 & 0.028 & 0.201 & 0.203 & 0.989 \\
\hline \multirow[t]{11}{*}{ I: } & GMM & -0.002 & 0.165 & 0.165 & 1.000 & 0.027 & 0.247 & 0.248 & 1.000 \\
\hline & FpGMM & -0.003 & 0.168 & 0.168 & 1.018 & 0.027 & 0.250 & 0.252 & 1.012 \\
\hline & FnpGMM & -0.003 & 0.168 & 0.168 & 1.014 & 0.027 & 0.250 & 0.251 & 1.010 \\
\hline & MGMM & -0.002 & 0.101 & 0.101 & 0.610 & 0.010 & 0.162 & 0.162 & 0.652 \\
\hline & FpMGMM & -0.003 & 0.116 & 0.116 & 0.701 & 0.013 & 0.180 & 0.180 & 0.725 \\
\hline & FnpMGMM & -0.007 & 0.171 & 0.172 & 1.038 & 0.028 & 0.253 & 0.255 & 1.026 \\
\hline & IV & -0.008 & 0.177 & 0.177 & 1.072 & 0.029 & 0.258 & 0.260 & 1.044 \\
\hline & OLS & -0.004 & 0.157 & 0.158 & 0.953 & 0.390 & 0.176 & 0.428 & 1.721 \\
\hline & WLS & 0.004 & 0.099 & 0.099 & 0.597 & 0.262 & 0.122 & 0.289 & 1.163 \\
\hline & FpWLS & 0.002 & 0.110 & 0.110 & 0.668 & 0.267 & 0.134 & 0.299 & 1.204 \\
\hline & FnpWLS & -0.007 & 0.170 & 0.170 & 1.028 & 0.048 & 0.241 & 0.245 & 0.988 \\
\hline
\end{tabular}




\begin{tabular}{|c|c|c|c|c|c|c|c|c|c|}
\hline \multirow{2}{*}{\multicolumn{2}{|c|}{ Table 5.4}} & \multicolumn{8}{|c|}{ Simulation results on coefficient estimates for cases J, K and L } \\
\hline & & \multicolumn{4}{|c|}{$\beta_{2}$} & \multicolumn{4}{|l|}{$\beta_{3}$} \\
\hline Case & & bias & st.dv & rmse & rrmse & bias & st.dv & rmse & rrmse \\
\hline \multirow[t]{11}{*}{$\mathrm{J}:$} & GMM & 0.002 & 0.078 & 0.078 & 1.000 & 0.020 & 0.207 & 0.207 & 1.000 \\
\hline & FpGMM & 0.002 & 0.078 & 0.078 & 1.002 & 0.019 & 0.207 & 0.208 & 1.002 \\
\hline & FnpGMM & 0.002 & 0.078 & 0.078 & 1.005 & 0.019 & 0.208 & 0.209 & 1.007 \\
\hline & MGMM & 0.001 & 0.055 & 0.055 & 0.708 & 0.011 & 0.153 & 0.153 & 0.740 \\
\hline & FpMGMM & 0.001 & 0.058 & 0.058 & 0.750 & 0.012 & 0.160 & 0.161 & 0.776 \\
\hline & FnpMGMM & 0.001 & 0.079 & 0.079 & 1.015 & 0.020 & 0.208 & 0.209 & 1.009 \\
\hline & IV & 0.001 & 0.079 & 0.079 & 1.017 & 0.020 & 0.208 & 0.209 & 1.008 \\
\hline & OLS & 0.001 & 0.069 & 0.069 & 0.889 & 0.465 & 0.079 & 0.472 & 2.276 \\
\hline & WLS & 0.002 & 0.051 & 0.051 & 0.652 & 0.428 & 0.062 & 0.433 & 2.085 \\
\hline & FpWLS & 0.002 & 0.053 & 0.053 & 0.686 & 0.426 & 0.065 & 0.431 & 2.079 \\
\hline & FnpWLS & 0.001 & 0.078 & 0.078 & 1.009 & 0.028 & 0.203 & 0.205 & 0.988 \\
\hline \multirow[t]{11}{*}{$\mathrm{K}:$} & GMM & 0.002 & 0.074 & 0.074 & 1.000 & 0.019 & 0.203 & 0.204 & 1.000 \\
\hline & FpGMM & 0.001 & 0.074 & 0.074 & 1.001 & 0.019 & 0.203 & 0.204 & 1.001 \\
\hline & FnpGMM & 0.001 & 0.075 & 0.075 & 1.007 & 0.019 & 0.205 & 0.206 & 1.010 \\
\hline & MGMM & 0.001 & 0.063 & 0.063 & 0.843 & 0.014 & 0.176 & 0.176 & 0.863 \\
\hline & FpMGMM & 0.001 & 0.067 & 0.067 & 0.902 & 0.016 & 0.186 & 0.187 & 0.914 \\
\hline & FnpMGMM & 0.001 & 0.075 & 0.075 & 1.009 & 0.019 & 0.204 & 0.205 & 1.004 \\
\hline & IV & 0.001 & 0.074 & 0.074 & 1.004 & 0.019 & 0.204 & 0.205 & 1.003 \\
\hline & OLS & 0.001 & 0.065 & 0.065 & 0.880 & 0.466 & 0.071 & 0.471 & 2.309 \\
\hline & WLS & 0.001 & 0.056 & 0.056 & 0.757 & 0.449 & 0.063 & 0.454 & 2.223 \\
\hline & FpWLS & 0.001 & 0.060 & 0.060 & 0.805 & 0.447 & 0.066 & 0.452 & 2.216 \\
\hline & FnpWLS & 0.001 & 0.074 & 0.074 & 1.001 & 0.027 & 0.198 & 0.200 & 0.982 \\
\hline \multirow[t]{11}{*}{$\mathrm{L}:$} & GMM & 0.001 & 0.071 & 0.071 & 1.000 & 0.018 & 0.200 & 0.201 & 1.000 \\
\hline & FpGMM & 0.001 & 0.071 & 0.071 & 1.000 & 0.018 & 0.201 & 0.201 & 1.001 \\
\hline & FnpGMM & 0.001 & 0.072 & 0.072 & 1.007 & 0.018 & 0.203 & 0.204 & 1.012 \\
\hline & MGMM & 0.001 & 0.068 & 0.068 & 0.957 & 0.017 & 0.193 & 0.194 & 0.962 \\
\hline & FpMGMM & 0.001 & 0.074 & 0.074 & 1.035 & 0.018 & 0.207 & 0.207 & 1.030 \\
\hline & FnpMGMM & 0.001 & 0.072 & 0.072 & 1.007 & 0.019 & 0.201 & 0.202 & 1.005 \\
\hline & IV & 0.001 & 0.071 & 0.071 & 1.000 & 0.018 & 0.201 & 0.201 & 1.001 \\
\hline & OLS & 0.001 & 0.063 & 0.063 & 0.879 & 0.466 & 0.065 & 0.471 & 2.339 \\
\hline & WLS & 0.001 & 0.060 & 0.060 & 0.847 & 0.461 & 0.063 & 0.466 & 2.314 \\
\hline & FpWLS & 0.001 & 0.065 & 0.065 & 0.910 & 0.459 & 0.067 & 0.464 & 2.307 \\
\hline & FnpWLS & 0.001 & 0.071 & 0.071 & 1.000 & 0.027 & 0.196 & 0.198 & 0.983 \\
\hline
\end{tabular}

In Table 5.4 we examine cases that differ from $\mathrm{B}$ only in that $0 \leq \lambda<1$, hence the heteroskedasticity does also depend now on a factor that one cannot capture in a feasible parametric technique. In case $\mathrm{J} \lambda=0.5$, in $\mathrm{K}$ it is 0.2 and $\lambda=0$ in case $\mathrm{L}$. In the latter case, where the heteroskedasticity is not related to any of the instruments, we note that the bias and variance results for GMM and IV are almost similar. This is due to

$$
\begin{aligned}
\operatorname{plim} n^{-1} Z^{\prime} \Omega Z & =\operatorname{plim} n^{-1} \sum_{i=1}^{n} \omega_{i} z_{i} z_{i}^{\prime} \\
& =\operatorname{plim} n^{-1} \sum_{i=1}^{n} z_{i} z_{i}^{\prime}+\operatorname{plim} n^{-1} \sum_{i=1}^{n}\left(\omega_{i}-1\right) z_{i} z_{i}^{\prime}=\operatorname{plim} n^{-1} Z^{\prime} Z
\end{aligned}
$$


because here $\omega_{i}=\exp \left(-\phi^{2} / 2+\phi \eta_{i}^{\circ}\right)$ with $E\left(\omega_{i} \mid z_{i}\right)=1$, thus the law of large numbers implies

$$
\operatorname{plim} n^{-1} \sum_{i=1}^{n}\left(\omega_{i}-1\right) z_{i} z_{i}^{\prime}=\lim n^{-1} \sum_{i=1}^{n} E\left[E\left(\omega_{i}-1 \mid z_{i}\right) z_{i} z_{i}^{\prime}\right]=O .
$$

A similar result yields the asymptotic equivalence of the standard and the heteroskedasticity consistent OLS variance estimators in models with just exogenous regressors which are unrelated with the disturbance variance. However, even for $\lambda=0$, MGMM beats GMM and IV. That MGMM does not converge to IV in this case follows from

$$
\begin{aligned}
\operatorname{plim} n^{-1} X^{\prime} \Omega^{-1} Z & =\operatorname{plim} n^{-1} \sum_{i=1}^{n} \omega_{i}^{-1} x_{i} z_{i}^{\prime} \\
& =\operatorname{plim} n^{-1} \sum_{i=1}^{n} x_{i} z_{i}^{\prime}+\operatorname{plim} n^{-1} \sum_{i=1}^{n}\left(\omega_{i}^{-1}-1\right) x_{i} z_{i}^{\prime} \\
& =\operatorname{plim} n^{-1} X^{\prime} Z+\operatorname{plim} n^{-1} \sum_{i=1}^{n} E\left[E\left(\omega_{i}^{-1}-1 \mid z_{i}\right) x_{i} z_{i}^{\prime}\right] \\
& \neq \operatorname{plim} n^{-1} X^{\prime} Z
\end{aligned}
$$

because $E\left(\omega_{i}^{-1}\right) \neq 1 / E\left(\omega_{i}\right)=1$. The results on the feasible MGMM estimators does reveal, however, that we have not managed yet to materialize this remarkable theoretical and experimental superiority of unfeasible MGMM for case L in a feasible implementation.

Next we examine the estimated standard errors. In Tables 5.5 through 5.7 for both $\beta_{2}$ and $\beta_{3}$ first the Monte Carlo estimate of the true standard deviation is repeated and next the average over the replications of the square root of the variance estimators given at the end of section 3 (indicated as st.er) are presented, followed by their ratio, which directly indicates the degree of over or under assessment of the true standard deviation.

In Table 5.5 we find for cases A through $\mathrm{C}$ that standard unfeasible GMM (substituting $\Omega$, but estimating $\sigma_{\varepsilon}^{2}$ ) is reasonable for strong instruments, but is more and more too pessimistic when instruments get weaker. This problem is primarily due to the estimation of $\sigma_{\varepsilon}^{2}$. In additional simulations not presented here we found similar results when using estimator $\hat{\sigma}_{\varepsilon}^{* 2}=\left(y^{*}-X^{*} \hat{\beta}_{G M M}\right)^{\prime}\left(y^{*}-X^{*} \hat{\beta}_{G M M}\right) / n$, whereas using the true value gave reasonable results for $\mu^{2} \geq 10$. On the other hand, the parametric implementation $\widehat{\operatorname{Var}}\left(\hat{\beta}_{F p G M M}\right)$ is much too optimistic, irrespective of the strength of the instruments, whereas the nonparametric implementation does not seem all that bad in this respect. Unfeasible MGMM is fine, but its feasible parametric version is as bad as it is for GMM, and as we already suspected in section 4 its examined nonparametric implementation is forlorn. The standard IV implementation, which neglects the heteroskedasticity completely, is found to be too optimistic. The variant which aims to repair this by employing a parametrically robustified variance estimate ( $\mathrm{VpIV}$ ) is even worse, whereas its nonparametric alternative (VnpIV) works remarkably well. Finally, we examine FnpWLS, which in Tables 5.1 through 5.4 kept up so remarkably well with GMM. We learn that a straight-forward nonparametric implementation of its variance estimate is extremely bad and overoptimistic. Table 5.5 also contains case D (mild heteroskedasticity). This shows again that FnpMGMM and FnpWLS should be discarded. It also illustrates again that operational FnpGMM is less vulnerable for weak instruments than unfeasible GMM, but that in fact FnpIV is not inferior to FnpGMM. Also when heteroskedasticity is mild there is yet no feasible variant for the superior estimator MGMM for which its standard errors are on average reasonably accurate for its true standard deviation. 


\begin{tabular}{|c|c|c|c|c|c|c|c|}
\hline \multirow{3}{*}{$\begin{array}{c}\text { Table } 5.5 \\
\text { Case }\end{array}$} & \multicolumn{7}{|c|}{ Simulation results on standard errors, cases A, B, C, D } \\
\hline & & \multicolumn{3}{|c|}{$\beta_{2}$} & \multicolumn{3}{|c|}{$\beta_{3}$} \\
\hline & & st.dv & st.er & ratio & st.dv & st.er & ratio \\
\hline \multirow[t]{10}{*}{ A: } & GMM & 0.081 & 0.084 & 1.038 & 0.115 & 0.117 & 1.019 \\
\hline & FpGMM & 0.082 & 0.036 & 0.434 & 0.115 & 0.066 & 0.573 \\
\hline & FnpGMM & 0.081 & 0.078 & 0.959 & 0.114 & 0.109 & 0.951 \\
\hline & MGMM & 0.045 & 0.044 & 0.993 & 0.068 & 0.067 & 0.996 \\
\hline & FpMGMM & 0.047 & 0.024 & 0.522 & 0.070 & 0.037 & 0.526 \\
\hline & FnpMGMM & 0.085 & 0.006 & 0.069 & 0.117 & 0.008 & 0.072 \\
\hline & IV & 0.086 & 0.071 & 0.828 & 0.118 & 0.108 & 0.915 \\
\hline & VpIV & 0.086 & 0.046 & 0.533 & 0.118 & 0.062 & 0.526 \\
\hline & VnpIV & 0.086 & 0.084 & 0.976 & 0.118 & 0.114 & 0.966 \\
\hline & FnpWLS & 0.085 & 0.006 & 0.066 & 0.116 & 0.007 & 0.058 \\
\hline \multirow[t]{10}{*}{ B: } & GMM & 0.082 & 0.100 & 1.218 & 0.211 & 0.246 & 1.170 \\
\hline & FpGMM & 0.082 & 0.034 & 0.415 & 0.211 & 0.119 & 0.562 \\
\hline & FnpGMM & 0.082 & 0.079 & 0.969 & 0.211 & 0.199 & 0.945 \\
\hline & MGMM & 0.045 & 0.045 & 0.997 & 0.123 & 0.123 & 0.998 \\
\hline & FpMGMM & 0.047 & 0.025 & 0.526 & 0.127 & 0.067 & 0.529 \\
\hline & FnpMGMM & 0.085 & 0.006 & 0.076 & 0.214 & 0.014 & 0.063 \\
\hline & IV & 0.086 & 0.072 & 0.843 & 0.215 & 0.197 & 0.917 \\
\hline & VpIV & 0.086 & 0.046 & 0.540 & 0.215 & 0.113 & 0.527 \\
\hline & VnpIV & 0.086 & 0.085 & 0.988 & 0.215 & 0.208 & 0.966 \\
\hline & FnpWLS & 0.085 & 0.006 & 0.066 & 0.209 & 0.008 & 0.039 \\
\hline \multirow[t]{10}{*}{$\mathrm{C}:$} & GMM & 0.089 & 0.131 & 1.475 & 0.528 & 0.628 & 1.189 \\
\hline & FpGMM & 0.089 & 0.039 & 0.438 & 0.531 & 0.256 & 0.483 \\
\hline & FnpGMM & 0.091 & 0.089 & 0.986 & 0.553 & 0.468 & 0.846 \\
\hline & MGMM & 0.046 & 0.046 & 1.017 & 0.268 & 0.266 & 0.993 \\
\hline & FpMGMM & 0.047 & 0.026 & 0.546 & 0.276 & 0.147 & 0.534 \\
\hline & FnpMGMM & 0.091 & 0.007 & 0.079 & 0.529 & 0.020 & 0.038 \\
\hline & IV & 0.091 & 0.083 & 0.912 & 0.528 & 0.464 & 0.879 \\
\hline & VpIV & 0.091 & 0.052 & 0.570 & 0.528 & 0.268 & 0.509 \\
\hline & VnpIV & 0.091 & 0.095 & 1.037 & 0.528 & 0.488 & 0.925 \\
\hline & FnpWLS & 0.087 & 0.006 & 0.067 & 0.442 & 0.010 & 0.022 \\
\hline \multirow[t]{10}{*}{ D: } & GMM & 0.075 & 0.092 & 1.224 & 0.204 & 0.240 & 1.176 \\
\hline & FpGMM & 0.075 & 0.035 & 0.462 & 0.204 & 0.119 & 0.582 \\
\hline & FnpGMM & 0.075 & 0.074 & 0.983 & 0.206 & 0.197 & 0.956 \\
\hline & MGMM & 0.063 & 0.064 & 1.011 & 0.176 & 0.175 & 0.994 \\
\hline & FpMGMM & 0.065 & 0.034 & 0.519 & 0.182 & 0.093 & 0.512 \\
\hline & FnpMGMM & 0.076 & 0.008 & 0.110 & 0.206 & 0.015 & 0.075 \\
\hline & IV & 0.075 & 0.072 & 0.961 & 0.205 & 0.198 & 0.967 \\
\hline & VpIV & 0.075 & 0.042 & 0.552 & 0.205 & 0.109 & 0.533 \\
\hline & VnpIV & 0.075 & 0.076 & 1.003 & 0.205 & 0.200 & 0.978 \\
\hline & FnpWLS & 0.075 & 0.007 & 0.094 & 0.200 & 0.009 & 0.043 \\
\hline
\end{tabular}




\begin{tabular}{|c|c|c|c|c|c|c|c|}
\hline \multirow[t]{3}{*}{ 1aide 5.0} & \multicolumn{7}{|c|}{ Simulation results on standard errors, cases E, F, G, H } \\
\hline & & \multicolumn{3}{|c|}{$\beta_{2}$} & \multicolumn{3}{|c|}{$\beta_{3}$} \\
\hline & & st.dv & st.er & ratio & st.dv & st.er & ratio \\
\hline \multirow[t]{8}{*}{ E: } & GMM & 0.083 & 0.083 & 1.004 & 0.211 & 0.205 & 0.974 \\
\hline & FpGMM & 0.083 & 0.035 & 0.421 & 0.211 & 0.127 & 0.601 \\
\hline & FnpGMM & 0.083 & 0.080 & 0.971 & 0.211 & 0.200 & 0.950 \\
\hline & MGMM & 0.045 & 0.045 & 0.993 & 0.124 & 0.123 & 0.990 \\
\hline & FpMGMM & 0.047 & 0.025 & 0.528 & 0.128 & 0.068 & 0.531 \\
\hline & IV & 0.087 & 0.074 & 0.844 & 0.216 & 0.198 & 0.920 \\
\hline & VpIV & 0.087 & 0.047 & 0.540 & 0.216 & 0.114 & 0.528 \\
\hline & VnpIV & 0.086 & 0.086 & 0.988 & 0.216 & 0.209 & 0.970 \\
\hline \multirow[t]{8}{*}{ F: } & GMM & 0.280 & 0.328 & 1.169 & 0.351 & 0.410 & 1.170 \\
\hline & FpGMM & 0.281 & 0.167 & 0.595 & 0.352 & 0.198 & 0.562 \\
\hline & FnpGMM & 0.280 & 0.266 & 0.949 & 0.351 & 0.332 & 0.945 \\
\hline & MGMM & 0.169 & 0.169 & 0.998 & 0.205 & 0.204 & 0.998 \\
\hline & FpMGMM & 0.175 & 0.093 & 0.528 & 0.212 & 0.112 & 0.529 \\
\hline & IV & 0.284 & 0.272 & 0.960 & 0.359 & 0.329 & 0.917 \\
\hline & VpIV & 0.284 & 0.150 & 0.528 & 0.359 & 0.189 & 0.527 \\
\hline & VnpIV & 0.284 & 0.275 & 0.969 & 0.359 & 0.346 & 0.966 \\
\hline \multirow[t]{8}{*}{ G: } & GMM & 0.075 & 0.098 & 1.309 & 0.215 & 0.270 & 1.255 \\
\hline & FpGMM & 0.075 & 0.033 & 0.445 & 0.216 & 0.120 & 0.558 \\
\hline & FnpGMM & 0.075 & 0.073 & 0.978 & 0.215 & 0.203 & 0.944 \\
\hline & MGMM & 0.044 & 0.044 & 0.999 & 0.123 & 0.123 & 0.999 \\
\hline & FpMGMM & 0.046 & 0.024 & 0.530 & 0.127 & 0.067 & 0.530 \\
\hline & IV & 0.077 & 0.072 & 0.932 & 0.223 & 0.197 & 0.885 \\
\hline & VpIV & 0.077 & 0.042 & 0.546 & 0.223 & 0.117 & 0.526 \\
\hline & VnpIV & 0.077 & 0.077 & 0.996 & 0.223 & 0.215 & 0.964 \\
\hline \multirow[t]{8}{*}{ H: } & GMM & 0.091 & 0.103 & 1.136 & 0.204 & 0.224 & 1.096 \\
\hline & FpGMM & 0.091 & 0.038 & 0.411 & 0.205 & 0.116 & 0.569 \\
\hline & FnpGMM & 0.091 & 0.087 & 0.959 & 0.204 & 0.194 & 0.951 \\
\hline & MGMM & 0.045 & 0.045 & 0.994 & 0.123 & 0.122 & 0.996 \\
\hline & FpMGMM & 0.047 & 0.025 & 0.523 & 0.127 & 0.067 & 0.529 \\
\hline & IV & 0.094 & 0.073 & 0.774 & 0.206 & 0.197 & 0.957 \\
\hline & VpIV & 0.094 & 0.050 & 0.534 & 0.206 & 0.109 & 0.529 \\
\hline & VnpIV & 0.094 & 0.092 & 0.981 & 0.206 & 0.200 & 0.972 \\
\hline
\end{tabular}

In Table 5.6 case E learns that also under mild simultaneity the parametric feasible variants estimate their variance poorly. Cases F, G and H support the wider validity of the earlier conclusions.

In Table 5.7 case I illustrates that all techniques tend more towards too optimistic standard error estimates when the sample size is smaller. From cases J through L we learn that the qualities of standard error estimates depend very little on the value of $\lambda$, except for IV. Note that for $\lambda=0$ (instruments and heteroskedasticity unrelated) the asymptotic equivalence of GMM and IV already shows up at $n=200$ for the coefficient estimates, but less so for their variance estimators. 


\begin{tabular}{|c|c|c|c|c|c|c|c|}
\hline \multirow{3}{*}{$\begin{array}{c}\text { Table } 5.7 \\
\text { Case }\end{array}$} & \multicolumn{7}{|c|}{ Simulation results on standard errors, cases I, J, K, L } \\
\hline & & \multicolumn{3}{|c|}{$\beta_{2}$} & \multicolumn{3}{|c|}{$\beta_{3}$} \\
\hline & & st.dv & st.er & ratio & st.dv & st.er & ratio \\
\hline \multirow[t]{8}{*}{ I: } & GMM & 0.165 & 0.157 & 0.953 & 0.247 & 0.231 & 0.935 \\
\hline & FpGMM & 0.168 & 0.064 & 0.381 & 0.250 & 0.118 & 0.473 \\
\hline & FnpGMM & 0.168 & 0.142 & 0.846 & 0.250 & 0.214 & 0.858 \\
\hline & MGMM & 0.101 & 0.096 & 0.950 & 0.162 & 0.152 & 0.940 \\
\hline & FpMGMM & 0.116 & 0.055 & 0.475 & 0.180 & 0.087 & 0.487 \\
\hline & IV & 0.177 & 0.143 & 0.810 & 0.258 & 0.230 & 0.893 \\
\hline & VpIV & 0.177 & 0.094 & 0.529 & 0.258 & 0.137 & 0.530 \\
\hline & VnpIV & 0.177 & 0.157 & 0.886 & 0.258 & 0.232 & 0.901 \\
\hline \multirow[t]{8}{*}{$\mathrm{J}:$} & GMM & 0.078 & 0.096 & 1.238 & 0.207 & 0.246 & 1.190 \\
\hline & FpGMM & 0.078 & 0.035 & 0.444 & 0.207 & 0.116 & 0.558 \\
\hline & FnpGMM & 0.078 & 0.076 & 0.976 & 0.208 & 0.198 & 0.952 \\
\hline & MGMM & 0.055 & 0.055 & 1.007 & 0.153 & 0.152 & 0.995 \\
\hline & FpMGMM & 0.058 & 0.030 & 0.515 & 0.160 & 0.082 & 0.513 \\
\hline & IV & 0.079 & 0.072 & 0.915 & 0.208 & 0.198 & 0.950 \\
\hline & VpIV & 0.079 & 0.043 & 0.544 & 0.208 & 0.110 & 0.528 \\
\hline & VnpIV & 0.079 & 0.079 & 0.998 & 0.208 & 0.203 & 0.974 \\
\hline \multirow[t]{8}{*}{$\mathrm{K}:$} & GMM & 0.074 & 0.092 & 1.242 & 0.203 & 0.243 & 1.196 \\
\hline & FpGMM & 0.074 & 0.035 & 0.470 & 0.203 & 0.115 & 0.564 \\
\hline & FnpGMM & 0.075 & 0.073 & 0.983 & 0.205 & 0.196 & 0.957 \\
\hline & MGMM & 0.063 & 0.063 & 1.013 & 0.176 & 0.174 & 0.991 \\
\hline & FpMGMM & 0.067 & 0.034 & 0.510 & 0.186 & 0.094 & 0.504 \\
\hline & IV & 0.074 & 0.072 & 0.971 & 0.204 & 0.198 & 0.972 \\
\hline & VpIV & 0.074 & 0.041 & 0.544 & 0.204 & 0.107 & 0.526 \\
\hline & VnpIV & 0.074 & 0.075 & 1.004 & 0.204 & 0.199 & 0.979 \\
\hline \multirow{8}{*}{$\mathrm{L}:$} & GMM & 0.071 & 0.085 & 1.193 & 0.200 & 0.231 & 1.152 \\
\hline & FpGMM & 0.071 & 0.035 & 0.491 & 0.201 & 0.109 & 0.541 \\
\hline & FnpGMM & 0.072 & 0.071 & 0.989 & 0.203 & 0.195 & 0.960 \\
\hline & MGMM & 0.068 & 0.069 & 1.016 & 0.193 & 0.190 & 0.987 \\
\hline & FpMGMM & 0.074 & 0.037 & 0.506 & 0.207 & 0.102 & 0.496 \\
\hline & IV & 0.071 & 0.072 & 1.014 & 0.201 & 0.198 & 0.988 \\
\hline & VpIV & 0.071 & 0.039 & 0.542 & 0.201 & 0.105 & 0.524 \\
\hline & VnpIV & 0.071 & 0.072 & 1.007 & 0.201 & 0.197 & 0.981 \\
\hline
\end{tabular}

The major findings from these simulations are that if $\Omega$ were known MGMM would be much more attractive than GMM, uniformly over all designs examined, because it has smaller bias, much smaller true standard deviation and also its standard errors establish much more accurate estimates of its actual standard deviation. Moreover, it is found to be less vulnerable to weakness of the instruments chosen for the original model specification. However, although a feasible parametric implementation of MGMM is often almost as efficient, its standard asymptotic variance estimate is very seriously biased and underestimates its actual dispersion, whereas the efficiency gains over GMM are completely lost by a nonparametric feasible MGMM implementation. The drawback of 
inaccurate standard errors by a feasible parametric implementation also affects GMM, but here the nonparametric implementation will provide reasonably accurate standard errors, provided the sample is not too small and the instruments not too weak. However, simply sticking to IV estimation and using nonparametric heteroskedasticity robust standard errors (VnpIV) is almost equally effective as employing feasible nonparametric GMM, because the latter suffers from weakened instruments due to weighing the observations. In order to fully exploit in practice the impressive efficiency gains achieved by feasible parametric MGMM it is yet required to develop a more accurate assessment of its actual efficiency. We leave this topic for future research.

It is a bit of a mystery why the inconsistent coefficient estimator FnpWLS performs often similar and occasionally even better than GMM. Nevertheless, it should not be recommended, because it underestimates its standard deviation often by a factor 20 or worse.

\section{Empirical illustrations}

To illustrate our theoretical findings in practice, we first set out to extend the 2SLS and GMM comparison for an actual cross-section data set as presented in Wooldridge (2001) with operational MGMM findings. However, the wage equation analyzed in that study does not seem to be inflicted with much heteroskedasticity, so in such a situation IV, possibly using heteroskedasticity consistent variance estimates, is self-evidently the preferable technique. Heteroskedasticity seems evident in a data set stemming from Sander (1992) on the effect of women's schooling on fertility, also addressed in Wooldridge (2010, Problem 6.8, dataset fertil1). The sample comprises 1129 US women. We regressed number of kids on education, age, age-squared a racial dummy and dummies for regions (east, west, north-central) and types of agglomeration (town, small-city, farm, other rural) and an intercept and year dummies. Education could be endogenous and is instrumented by years of education of the father and of the mother. Hence, the degree of overidentification is just one. The $F$-test on the exclusion of the two external instruments in the reduced form equation for education is 155.8 so they are certainly not weak. However, the endogeneity problem does not seem severe. The DWH statistic has p-value 0.48 , so many researchers would happily accept exogeneity of all regressors. However, the power of such a test is not always impressive, see Kiviet and Pleus (2014), so imposing exogeneity could be rash, especially because there are good theoretical reasons to assume endogeneity of years of schooling. Some mothers may have yet relatively few years of education because they already care for children. This would explain the positive difference between the OLS and IV coefficient estimates. That positive difference may also be the result of omitted control variables which have a positive effect on fertility and are negatively correlated with years of education (or vice versa, such as, for instance, being aware of birth control methods). Classic tests for heteroskedasticity after OLS estimation are all highly significant. Employing the instruments in the various MM techniques examined here does not provide any evidence of instrument invalidity by the Sargan-Hansen $J$ test, as can be seen from Table 6.1 , which does not mention the results for the included demographic and year-dummy controls.

The parametric feasible results in Table 6.1 have been obtained as follows. We examined the log of the squared IV residuals. These have skewness 0.26 and kurtosis 3.98. Hence, although they are significantly nonnormal, their distribution is not completely 
out of line with those of $g_{i}$ in (4.8). Their sample mean is -0.47 and sample variance 0.66. Interpreting these as reflecting the expectation and variance of $g_{i}$ they would imply $\phi$ to be 0.69 or 0.81 respectively. Next we ran a regression of the log of the squared IV residuals on all the instruments, their squares and their cross-products, leaving out redundant contributions such as the squares of dummies and the cross-products of two annual dummies, of two regional dummies and of two agglomeration dummies (giving 149 regressors in addition to the constant). This regression yields an $R^{2}$ of only 0.128 ; this we associate with a value of $\lambda$ in (4.8) as low as about 0.13. Although some individual coefficients in this auxiliary regression have substantial $t$-ratio's the overall $F$-test has $p$-value 0.61. So, one could conclude that heteroskedasticity determined by the instrumental variables is insignificant (although in a more parsimonious specification significant heteroskedasticity would emerge). We obtained a series of positive $\hat{\sigma}_{i}$ values by taking the exponential function of the fitted values of this (unrestricted) auxiliary regression. Next, by dividing these by their sample average we obtained a series $\hat{\omega}_{i}$ with sample average unity. This series has empirical quantiles $q^{0.005}\left(\hat{\omega}_{i}\right)=0.094$ and $q^{0.995}\left(\hat{\omega}_{i}\right)=7.970$, which matches with a $\phi$ in the range $0.8-1.0$ according to Table 4.1.

\begin{tabular}{|c|c|c|c|c|c|c|c|}
\hline le 6.1 & \multicolumn{7}{|c|}{ Empirical findings on fertility (standard errors between parentheses) } \\
\hline & OLS & IV & $\mathrm{IV}(\mathrm{Vnp})$ & FpGMM & FnpGMM & FpMGMM & FnpMGMM \\
\hline educ & $\begin{array}{l}-0.128 \\
(0.018)\end{array}$ & $\begin{array}{l}-0.153 \\
(0.039)\end{array}$ & $\begin{array}{l}-0.153 \\
(0.041)\end{array}$ & $\begin{array}{l}-0.152 \\
(0.050)\end{array}$ & $\begin{array}{l}-0.153 \\
(0.041)\end{array}$ & $\begin{array}{l}-0.168 \\
(0.033)\end{array}$ & $\begin{array}{l}-0.155 \\
(0.003)\end{array}$ \\
\hline age & $\begin{array}{c}0.532 \\
(0.138)\end{array}$ & $\begin{array}{c}0.524 \\
(0.139)\end{array}$ & $\begin{array}{c}0.524 \\
(0.141)\end{array}$ & $\begin{array}{c}0.523 \\
(0.183)\end{array}$ & $\begin{array}{c}0.523 \\
(0.141)\end{array}$ & $\begin{array}{c}0.421 \\
(0.127)\end{array}$ & $\begin{array}{c}0.524 \\
(0.013)\end{array}$ \\
\hline agesq & $\begin{array}{r}-0.006 \\
(.002)\end{array}$ & $\begin{array}{l}-0.006 \\
(0.002)\end{array}$ & $\begin{array}{l}-0.006 \\
(0.002)\end{array}$ & $\begin{array}{l}-0.006 \\
(0.002)\end{array}$ & $\begin{array}{l}-0.006 \\
(0.002)\end{array}$ & $\begin{array}{l}-0.005 \\
(0.001)\end{array}$ & $\begin{array}{l}-0.006 \\
(0.000)\end{array}$ \\
\hline black & $\begin{array}{c}1.076 \\
(0.174) \\
\end{array}$ & $\begin{array}{c}1.073 \\
(0.174)\end{array}$ & $\begin{array}{c}1.073 \\
(0.201)\end{array}$ & $\begin{array}{c}1.068 \\
(0.301)\end{array}$ & $\begin{array}{c}1.072 \\
(0.201)\end{array}$ & $\begin{array}{c}0.881 \\
(0.177)\end{array}$ & $\begin{array}{c}1.129 \\
(0.032)\end{array}$ \\
\hline$J$ (p-val.) & - & 0.88 & 0.88 & 0.91 & 0.88 & 0.90 & 0.70 \\
\hline
\end{tabular}

Given our findings regarding $\phi, \lambda$ and the strength of the instruments these empirical results can probably best be interpreted against the background of our simulation results for cases $\mathrm{A}$ and $\mathrm{K}$, although the sample size in this application is much larger. In the simulation the cases $A$ and $K$ suggest similar performance regarding rmse by IV and the two feasible GMM techniques, but serious understatement by FpGMM of its actual standard deviation, and much less so by IV. However, the empirical results show substantially smaller estimated standard errors for FnpGMM than for FpGMM. We have no simple explanation for this. We should keep in mind though that in this empirical example the number of included instruments in comparison to the number of excluded instruments is completely different from the simulation design. Moreover, having just overidentification of order 1 makes interpretation of standard error estimates difficult anyhow, because formally the coefficient variance does not exist. Also, the patterns in the Tables 5.5 through 5.7 are about the expectation of standard error estimates, and not about single random realizations as obtained in Table 6.1. Nevertheless, the similarity in 
the standard error results for FnpGMM and the nonparametrically robustified standard errors of IV, which are slightly larger than the (incorrect) standard IV standard errors, is in agreement with our simulation findings.

Although we should always realize that a difference in empirical standard errors does not necessarily represent a similar difference in true standard deviations, the most remarkable finding from Table 6.1 is undoubtedly that the standard errors of MGMM are in agreement with the in the simulations established superiority of FpMGMM over FpGMM and FnpGMM (when $\lambda>0$ ). The results in the final column demonstrate that, although the coefficient estimates of FnpMGMM are not inferior to GMM, this technique is unable to assess its own accuracy, which it extremely seriously overrates.

We also employed the various techniques to data analyzed in Wu et al. (2014). We reanalyzed one of its two structural equations for land and house prices using data for 2011 on the 35 major Chinese cities. Table 6.2 presents the effect of house price (hp) on land price, but does not mention results on further control variables, such as lagged budget deficit, construction costs, agricultural GDP and available land. External instruments used are disposable income, total population, sex ratio and expenditure on education, giving a degree of overidentification of 4 . The DWH statistic has $p$-value 0.043 , the 2SLS results yield a $J$-statistic with a $p$-value of 0.40 , but in the reduced form equation the external instruments produce an $F$-value of only 6.87 . Hence, it seems that house prices are endogenous and the employed instruments are valid though weak, although such a small sample of course hardly allows firm inferences of this nature. The auxiliary regression of the log of the squared 2SLS residuals on all 10 instruments yields an $R^{2}$ of 0.21 (we left out squares and cross-products because that would slurp all remaining degrees of freedom). From its fitted values we obtained a series for $\hat{\omega}_{i}$ as before.

\begin{tabular}{lccccccc}
\hline Table 6.2 & \multicolumn{6}{c}{ Empirical findings on land prices (standard errors between parentheses) } \\
\hline & OLS & IV & IV(Vnp) & FpGMM & FnpGMM & FpMGMM & FnpMGMM \\
\hline $\mathrm{hp}$ & 1.179 & 1.706 & 1.706 & 1.638 & 1.321 & 1.638 & 1.507 \\
& $(0.309)$ & $(0.426)$ & $(0.461)$ & $(0.352)$ & $(0.476)$ & $(0.317)$ & $(0.145)$ \\
\hline$J$ (p-val.) & - & 0.40 & 0.40 & 0.60 & 0.31 & 0.72 & 0.80 \\
\hline
\end{tabular}

Again we note the misrepresentation by FnpMGMM of its precision, but also the attractive standard errors of FpMGMM.

\section{Conclusions}

We reveal an inherent unfavorable and yet generally unperceived feature of GMM as it is currently usually implemented. Extracting from the assumed orthogonality conditions instrumental variables such that they are reasonably effective (strong) for the regressors in the habitual sense, as understood for IV estimation, implies that these very same instruments will be much weaker in the context of GMM. This is because, implicitly, GMM estimates a transformed model, in order to get rid of any non-sphericity of the disturbances, but at the same time this transformation affects the instruments in such a way that they will actually be much weaker than the researcher realizes. It is shown, however, that relatively simple precautions enable to neutralize this weakening process 
of the instruments. This also allows to improve on IV and GMM in just identified models with heteroskedasticity.

By simulation it is shown that empirically relevant forms of heteroskedasticity undermine the quality of standard GMM estimates and a modified implementation of GMM yields estimates that show both less bias and smaller standard deviations. Reductions of the root mean squared errors of the coefficient estimates of the alleged optimal standard GMM technique by a factor 2 or more are shown to be not exceptional when heteroskedasticity is prominent. We also examine the accuracy of empirical standard errors for the underlying true standard deviations.

In this paper we only examined GMM estimators for cross-sectional models that are linear in the regressor coefficients. However, the results have implications for general nonlinear models too, and also for the analysis of time-series data and especially for dynamic panel data models where GMM is used frequently. Next to our simulation findings, we also examined for empirical data sets what the practical consequences are. Interpreting these, one should keep in mind, that the synthetic simulation experiments produce accurate assessments of true bias and true standard deviations, whereas for the empirical findings any bias cannot be assessed, because the true parameter values are unknown, and the obtained estimated standard errors may be very misleading for the underlying unknown true standard deviations. Nevertheless, we do find substantially smaller estimated standard errors and therefore we unreservedly recommend the use of parametric MGMM, the modified form of feasible GMM as developed here.

\section{References}

Andrews, D.W.K., Stock, J.H., 2007. Inference with Weak Instruments, Chapter 6 in: Blundell, R., Newey, W.K., Persson, T. (eds.), Advances in Economics and Econometrics, Theory and Applications, 9th Congress of the Econometric Society, Vol. 3. Cambridge, UK: Cambridge University Press.

Arellano, M., 2002. Sargan's instrumental variables estimation and the generalized method of moments. Journal of Business and Economic Statistics 20, 450-459.

Arellano, M., 2003a. Panel Data Econometrics. Oxford University Press: Advanced Texts in Econometrics, Oxford.

Arellano, M., 2003b. Modelling Optimal Instrumental Variables for Dynamic Panel Data Models". CEMFI Working Paper no. 0310.

Baltagi, B.H., Li, Q., 1992. A note on the estimation of simultaneous equations with error components. Econometric Theory 8, 113-119.

Bowden, J.B., Turkington, D.A., 1984. Instrumental Variables. Econometric Society Monograph in Quantitative Economics. Cambridge University Press.

Cameron, A.C., Trevedi, P.K., 2005. Microeconometrics: Methods and Applications. Cambridge University Press.

Davidson, R., MacKinnon, J.G., 2004. Econometric Theory and Methods. Oxford University Press.

Hall, A.R., 2005. Generalized Method of Moments. Oxford University Press.

Hansen, L.P., 1982. Large sample properties of generalized method of moments estimators. Econometrica 50, 1029-1054.

Hausman, J.A., Newey, W.K., Woutersen, T., Chao, J.C., Swanson, N.R., 2012. Instrumental variable estimation with heteroskedasticity and many instruments. Quan- 
titative Economics 3, 211-255.

Iglesias, E.M., Phillips, G.D.A., 2012. Improved instrumental variables estimation of simultaneous equations under conditionally heteroskedastic disturbances. Journal of Applied Econometrics 27, 474-499.

Kiviet, J.F., 2012. Monte Carlo Simulation for Econometricians. Foundations and Trends in Econometrics 5:1-2, 1-181. Now Publishers, Boston - Delft.

Kiviet, J.F., 2013. Identification and inference in a simultaneous equation under alternative information sets and sampling schemes. The Econometrics Journal 16, S24S59.

Kiviet, J.F., Pleus, M., 2014. The performance of tests on endogeneity of subsets of explanatory variables scanned by simulation. Mimeo.

Newey, K.W., 1993. Efficient estimation of models with conditional moment restrictions. Chapter 16 in: Handbook of Statistics, Volume 11, Econometrics (G.S. Maddala, C.R. Rao and H.D. Vinod, eds.), 419-454, Elsevier Science Publishers.

Sander, W., 1992. The effect of women's schooling on fertility. Economics Letters 40, 229-233.

Sargan, J.D., 1958. The estimation of economic relationships using instrumental variables. Econometrica 26, 393-415.

Sargan, J.D., 1959. The estimation of relationships with autocorrelated residuals by the use of instrumental variables. Journal of the Royal Statistical Society, Series B 21, 91-105.

Staiger, D., Stock, J.H., 1997. Instrumental variables regression with weak instruments. Econometrica 65, 557-586.

Wansbeek, T.W., 2004. Correcting for heteroskedasticity of unspeified form (Exercise 04.1.2). Econometric Theory 20, 224.

White, H., 1986. Instrumental variables analogs of generalized least squares estimators. In: Advances in Statistical Analysis and Statistical Computing Vol.1 (R.S. Mariano, ed.), 173-227. JAI Press.

Wooldridge, J.M., 2001. Applications of Genralized Method of Moments estimation. Journal of Economic Perspectives 15, 87-100.

Wooldridge, J.M., 2010. Econometric Analysis of Cross Section and Panel Data, second edition. The MIT Press, Cambridge USA.

Wooldridge, J.M., 2013. Introductory Econometrics, A Modern Approach, fifth edition. South-Western, Cengage Learning.

Wu, G.L., Feng, Q., Li, P., 2014. Does local governments' budget deficit pus up housing prices in China? Forthcoming in China Economic Review. EGC Report 2014/09, Nanyang Technological University, Singapore. 